\title{
Chemoselective synthesis of polyfunctional aminophenyl 2-oxobut-3-enyl - and quinolinylmethyl- $C$-glycopyranosides from nitrophenyl 2-oxobut-3-enyl $C$-glycopyranosides under ultrasonic vibration
}

\author{
K. Kumar G. Ramakrishna, Arya Ajay, Anindra Sharma, and Rama P. Tripathi* \\ Medicinal \& Process Chemistry Division, CSIR-Central Drug Research Institute, \\ P.O. Box 173, Chattar Manzil, Mahatma Gandhi Marg, Lucknow 226 001, India \\ E-mail: rp_tripathi@cdri.res.in, rpt.cdri@gmail.com
}

Dedicated to Prof. Richard R. Schmidt on the occasion of his $78^{\text {th }}$ birthday

\begin{abstract}
Chemoselective reduction of nitro group in polyfunctional nitrophenyl 2-oxobut-3-enyl $C$ glycopyranosides to the respective aminophenyl 2-oxobut-3-enyl glycopyranosides with $\mathrm{SnCl}_{2} \cdot 2 \mathrm{H}_{2} \mathrm{O}$ under ultrasonic vibration in good yields was achieved successfully. Other potentially reducible groups such as carbonyl, ester, azide, tosyl, alkenic substituents were unaffected during reaction. The 2'-nitrophenyl-2-oxobut-3-enyl glycopyranosides as reduction substrates gave 2-quinolinemethyl glycopyranosides via reduction followed by intramolecular cyclocondensation reactions. These $\beta$ - $C$-glycopyranosides hold great promise in medicinal chemistry.
\end{abstract}

Keywords: Chemoselective reduction, ultrasound sonicator, tin(II) reduction, polyfunctional $C$-glycopyranosides, quinolines

\section{Introduction}

Compounds with aniline moiety are key intermediates to the synthesis, inter alia, of dyes, herbicides, pesticides, and pharmaceuticals. ${ }^{1-4}$ The application of conventional catalytic systems to the selective reduction of a nitro group in nitroarenes in the presence of other potentially reducible functional groups, e.g., halogen, carbonyl, cyano, benzyloxy, tosyloxy, acetyl and alkenic groups has many drawbacks as the reaction is often accompanied by the reduction of other functional groups as well and sometimes reduction of benzene ring to its cyclohexyl counterpart resulting in complex reaction mixture. ${ }^{5-7}$ Redox-economical transformations are the 
key steps of chemical synthesis in nature for complex natural products. ${ }^{8-11}$ Aryl $C$ - $\beta$ glycopyranosides are key components in many naturally occurring antibiotics and are potent chemotherapeutic agents. ${ }^{12-17}$ The $C$-glycosidic bond in these compounds offers stability towards enzymatic and chemical hydrolysis and therefore several of them are potent inhibitors of glycosidases and glycosyl transferases. ${ }^{18-22}$ The aminoaryl glycopyranosides possess insulin-like activity and so have chemotherapeutic potential in diabetes, ${ }^{23}$ and therefore the new synthetic methods to access these compounds are still in great demand.

Organic syntheses with chemoselectivity are of immense importance in order to avoid unnecessary protection and deprotection steps and also to reduce the cost of overall synthetic processes. $^{24-27}$ The reaction of a particular functional group selectively in polyfunctional molecules is challenging. ${ }^{28}$ A number of methods for the reduction of a nitro group in the presence of other functional groups in nitroarenes have been reported with high levels of chemoselectivity. ${ }^{29-35}$ However, to the best of our knowledge the chemoselective reduction of aromatic nitro groups in nitroaryl glycosides is rare.

Herein, we report the ultrasound-mediated chemoselective reduction of aromatic nitro groups in polyfunctional nitrophenyl 2-oxobut-3-enyl glycopyranosides to the respective anilinyl 2oxobut-3-enyl- or 2-quinolinemethyl $\beta$-D-C-glycopyranosides using $\mathrm{SnCl}_{2} \cdot 2 \mathrm{H}_{2} \mathrm{O}$ under ambient reaction conditions. The application of the selected compounds of the series for the preparation of many biologically active compounds has also been investigated. Our method does not affect the other functional groups in the sugar moiety and the double bonds and ketone moieties are unchanged except in the case of the 2-nitrophenyl series.

\section{Results and Discussion}

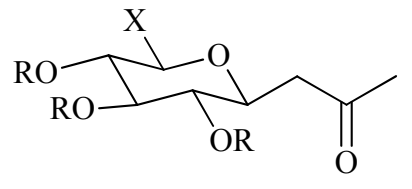

$\mathbf{X}=\mathrm{H}$ or $\mathrm{CH}_{2} \mathrm{OR}$

$\mathbf{R}=\mathrm{H}$ or $\mathrm{COCH}_{3}$ (i) $20 \mathrm{~mol} \%$ L-proline $/ \mathrm{Et}_{3} \mathrm{~N}$, $\mathrm{MeOH}$, nitrobenzaldehydes, $\mathrm{RT}, 30-40 \mathrm{~h}$, for $\mathrm{R}=\mathrm{H}$

(ii) $20 \mathrm{~mol} \%$ pyrrolidine, $\mathrm{CH}_{2} \mathrm{Cl}_{2}$, nitrobenzaldehydes, $\mathrm{RT}, 20-24 \mathrm{~h}$, for $\mathrm{R}=\mathrm{COCH}_{3}$

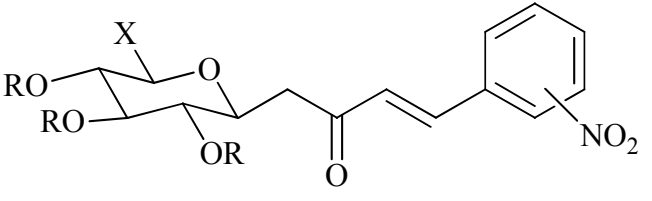

$1,2 a, 2 b, 3 a-3 c, 4 a-4 c$

\begin{tabular}{c|llll} 
Compd. No. & $\mathbf{R}$ & \multicolumn{1}{c}{$\mathbf{X}$} & Position of $\mathbf{N O}_{2}$ & Yield \% \\
\hline $\mathbf{1}$ & $\mathrm{H}$ & $\mathrm{H}$ & $2-\mathrm{NO}_{2}$ & 57 \\
$\mathbf{2 a}$ & $\mathrm{H}$ & $\mathrm{CH}_{2} \mathrm{OH}$ & $3-\mathrm{NO}_{2}$ & 68 \\
$\mathbf{2 b}$ & $\mathrm{H}$ & $\mathrm{CH}_{2} \mathrm{OH}$ & $4-\mathrm{NO}_{2}$ & 69 \\
$\mathbf{3 a}$ & $\mathrm{Ac}$ & $\mathrm{H}$ & $2-\mathrm{NO}_{2}$ & 65 \\
3b & $\mathrm{Ac}$ & $\mathrm{H}$ & $3-\mathrm{NO}_{2}$ & 65 \\
$\mathbf{3 c}$ & $\mathrm{Ac}$ & $\mathrm{H}$ & $4-\mathrm{NO}_{2}$ & 71 \\
$\mathbf{4 a}$ & $\mathrm{Ac}$ & $\mathrm{CH}_{2} \mathrm{OAc}$ & $2-\mathrm{NO}_{2}$ & 67 \\
$\mathbf{4 b}$ & $\mathrm{Ac}$ & $\mathrm{CH}_{2} \mathrm{OAc}$ & $3-\mathrm{NO}_{2}$ & 70 \\
4c & $\mathrm{Ac}$ & $\mathrm{CH}_{2} \mathrm{OAc}$ & $4-\mathrm{NO}_{2}$ & 72
\end{tabular}

Scheme 1. Synthesis of nitrophenyl 2-oxobut-3-enyl-1'-deoxy- $C$-glycopyranosides. 
The starting nitrophenyl 2-oxobut-3-enyl-1'-deoxy- $\beta$-D- $C$-glycopyranosides (1, 2a and 2b) and the peracetylated 2-oxobut-3-enyl-1'-deoxy- $\beta$-D- $C$-glycopyranosides $(\mathbf{3 a - 3 c}, 4 \mathbf{a}-\mathbf{4 c})$ were prepared from commercially available sugars D-xylose and D-glucose following earlier reported protocols. ${ }^{36-39}$ The spectroscopic data of these compounds are similar to those prepared earlier, ${ }^{36}$ and most of the newly synthesized compounds follow the same pattern.

To optimize the reaction conditions for the chemoselective reduction, a model substrate $(E)$ 4-(3-nitrophenyl)-1-[1'-deoxy-2',3',4',6'-tetra- $O$-acetyl- $\beta$-D-glucopyranos-1'-yl]but-3-en-2-one (4b) was reduced with different reducing agents using different experimental conditions under ultrasonic vibration at $30{ }^{\circ} \mathrm{C}$ to give the respective $(E)$-4-(3-aminophenyl)-1-[1'-deoxy-2',3',4',6'tetra- $O$-acetyl- $\beta$-D-glucopyranos-1'-yl]but-3-en-2-one $(\mathbf{8 b})$ in varying yields (Scheme 2 ).

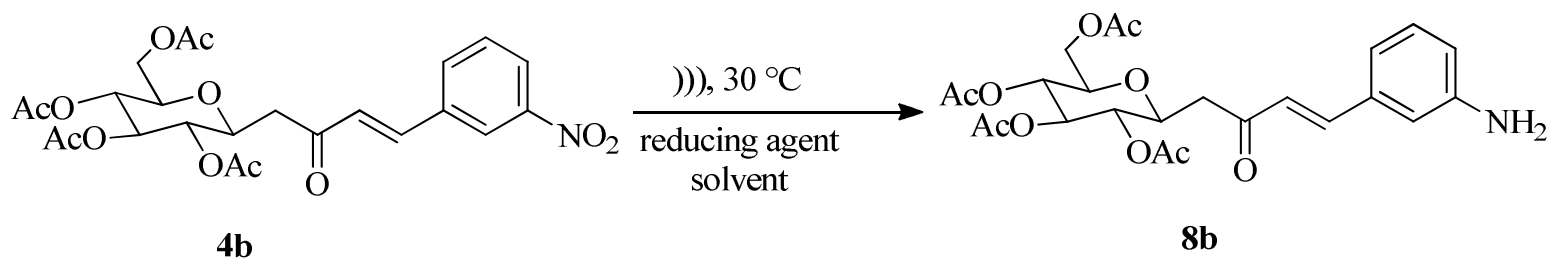

Scheme 2. A model chemoselective reduction of nitro phenyl 2-oxobut-3-enyl-1'-deoxyglucopyranoside (4b) in the presence of various reducing agents and solvents.

$\mathrm{SnCl}_{2} \cdot 2 \mathrm{H}_{2} \mathrm{O}$ and $\mathrm{Fe}(\mathrm{s}) / \mathrm{AcOH}$ were screened for reduction purpose in different solvents and the results are summarized in Table 1. The application of EtOH in the presence of $\mathrm{SnCl}_{2} \cdot 2 \mathrm{H}_{2} \mathrm{O}$ (10 eq.) at $30{ }^{\circ} \mathrm{C}$ in ultrasonic bath was found to be the most suitable condition to offer the maximum yield (68\%) of the desired compound $\mathbf{8 b}$. Increasing the load of catalyst does not affect the yield of the product, however the time for the completion of reaction is slightly reduced (Table 1, entry 4 and 5). Although the conventional stirring at $60{ }^{\circ} \mathrm{C}$ and in refluxing condition the yield of the desired product is comparable to that of ultrasonic bath yet the time required in conventional stirring is significantly enhanced (Table 1, entry $\mathbf{7}$ and $\mathbf{8}$ ). It is important to mention here that minor products (detected by TLC) formed during the reaction could not be isolated in pure form to be characterized.

The structural elucidation of compound $\mathbf{8 b}$ was carried out on the basis of its spectroscopic data. HRMS of the compound displays $m / z .514 .1662 \mathrm{amu}$ as $[\mathrm{M}+\mathrm{Na}]^{+}$peak corresponding to its molecular formulae $\mathrm{C}_{24} \mathrm{H}_{29} \mathrm{NO}_{10}$. In the ${ }^{1} \mathrm{H}$ NMR spectrum, the two exchangeable $\mathrm{NH}_{2}$ protons were observed at $\delta 3.72$ while the two olefinic protons were visible as doublets at $\delta 7.46(\mathrm{~d}, 1 \mathrm{H}$, $J=16.1 \mathrm{~Hz}, \mathrm{H}-4)$ and $\delta 6.68(\mathrm{~d}, 1 \mathrm{H}, J=16.1 \mathrm{~Hz}, \mathrm{H}-3)$ besides other usual protons at their usual chemical shift. In ${ }^{13} \mathrm{C}$ NMR spectrum a signal at $\delta 195.7$ accounted the ketonic group carbon $\left(\mathrm{CH}_{2}-(\mathrm{C}=\mathrm{O})-\mathrm{C}=\mathrm{C}-\right)$, quite distinct from the acetyl carbon signals at $\delta 170.2,169.8,169.5$ and 169.1. The olefinic carbon signals were visible at $\delta 144.0(\mathrm{C}-4)$ and $126.0(\mathrm{C}-3)$, while the methylene carbon of the alkenonyl moiety was observed at $\delta 42.6$ along with other usual signals. 
Table 1. Optimization of the chemoselective reduction of $(E)-4-(3-$ nitrophenyl)-1-[1'-deoxy$2^{\prime}, 3^{\prime}, 4^{\prime}, 6^{\prime}$-tetra- $O$-acetyl- $\beta$-D-glucopyranos-1'-yl]but-3-en-2-one(4b)

\begin{tabular}{|c|c|c|c|c|}
\hline Entry & Reducing agent & Solvent & $\begin{array}{c}\text { Reaction } \\
\text { time (min) }\end{array}$ & $\begin{array}{l}\text { Isolated } \\
\text { yield }(\%)\end{array}$ \\
\hline 1 & $\begin{array}{l}\mathrm{SnCl}_{2} \cdot 2 \mathrm{H}_{2} \mathrm{O} \\
\text { (1 equiv.) }\end{array}$ & $\mathrm{EtOH}$ & 360 & 28 \\
\hline 2 & $\begin{array}{l}\mathrm{SnCl}_{2} \cdot 2 \mathrm{H}_{2} \mathrm{O} \\
\text { (5 equiv.) }\end{array}$ & $\mathrm{EtOH}$ & 240 & 43 \\
\hline 3 & $\begin{array}{l}\mathrm{SnCl}_{2} \cdot 2 \mathrm{H}_{2} \mathrm{O} \\
\text { (10 equiv.) }\end{array}$ & $\mathrm{EtOH}$ & 120 & 68 \\
\hline 4 & $\begin{array}{c}\mathrm{SnCl}_{2} \cdot 2 \mathrm{H}_{2} \mathrm{O} \\
\text { (15 equiv.) }\end{array}$ & $\mathrm{EtOH}$ & 110 & 65 \\
\hline 5 & $\begin{array}{l}\mathrm{SnCl}_{2} \cdot 2 \mathrm{H}_{2} \mathrm{O} \\
\text { (20 equiv.) }\end{array}$ & $\mathrm{EtOH}$ & 105 & 66 \\
\hline 6 & $\begin{array}{c}\mathrm{SnCl}_{2} \cdot 2 \mathrm{H}_{2} \mathrm{O} \\
(10 \text { equiv., } \mathrm{RT})^{*}\end{array}$ & $\mathrm{EtOH}$ & 540 & 52 \\
\hline 7 & $\begin{array}{c}\mathrm{SnCl}_{2} .2 \mathrm{H}_{2} \mathrm{O} \\
\left(10 \text { equiv., } 60^{\circ} \mathrm{C}\right)^{*}\end{array}$ & $\mathrm{EtOH}$ & 240 & 63 \\
\hline 8 & $\begin{array}{c}\mathrm{SnCl}_{2} .2 \mathrm{H}_{2} \mathrm{O} \\
(10 \text { equiv., reflux })^{*}\end{array}$ & $\mathrm{EtOH}$ & 210 & 64 \\
\hline 9 & $\begin{array}{c}\mathrm{Fe}(\mathrm{s}) \\
\text { (1 equiv.) }\end{array}$ & $\begin{array}{c}\text { EtOH: } \mathrm{AcOH}: \\
\mathrm{H}_{2} \mathrm{O}\end{array}$ & 420 & 25 \\
\hline 10 & $\begin{array}{c}\mathrm{Fe}(\mathrm{s}) \\
\text { (5 equiv.) }\end{array}$ & $\begin{array}{c}\text { EtOH:AcOH: } \\
\mathrm{H}_{2} \mathrm{O}\end{array}$ & 240 & 52 \\
\hline 11 & $\begin{array}{c}\mathrm{Fe}(\mathrm{s}) \\
\text { (5 equiv.) }\end{array}$ & AcOH: $\mathrm{H}_{2} \mathrm{O}$ & 300 & 48 \\
\hline
\end{tabular}

Above all the reactions are conducted in ultrasonic bath expect entries 6-8 which were conducted under conventional stirring.

Having optimized reaction condition for the chemoselective reduction of the nitro group, its scope was investigated with other substrates also, where the reduction of the above nitro phenyl 2-oxobut-3-enyl-1'-deoxy-glycopyranosides $\mathbf{2 a}, \mathbf{2 b}, \mathbf{3 b}, \mathbf{3 c}, \mathbf{4 b}$ and $\mathbf{4 c}$ (except 2-nitro substituted systems, 1, 3a and $\mathbf{4 a}$ ) with 10 equiv. of $\mathrm{SnCl}_{2} \cdot 2 \mathrm{H}_{2} \mathrm{O}$ under ultrasonic vibration in ethanol at 30 ${ }^{\circ} \mathrm{C}$ separately led to the formation respective 4-(aminophenyl) -2-oxobut-3-enyl-1'-deoxyglycopyranosides $(\mathbf{6 a}, \mathbf{6 b}, \mathbf{7 b}, \mathbf{7 c}, \mathbf{8 b}$ and $\mathbf{8 c}$ ) in good yields (Scheme 3, Table 2). One of the interesting observations made during the reduction of the 4-(nitrophenyl)-2-oxobut-3-enyl-1'deoxy-glycopyranosides with or without protected hydroxyl groups in the sugar moiety was that all of them underwent smooth reduction of the nitro group in aromatic ring irrespective of the nature of the sugar without affecting other potentially reducible functional groups. To our pleasant surprise, reduction of the 4-(2-nitrophenyl)-2-oxobut-3-enyl-1'-deoxy- $\beta$-D-glyco- 
pyranosides (1, 3a and 4a) under above mentioned condition led to formation of products unexpectedly with almost same $R_{\mathrm{f}}$ values as the starting material but devoid of the nitro and carbonyl groups. The compounds were isolated and characterized as quinolin-2-methyl glycopyranosides (5, 7a and 8a) in good yields (Scheme 3, Table 2). Such observations were earlier reported by Silva's group ${ }^{40,41}$ during reduction of 2-nitro chalcone with $\mathrm{SnCl}_{2}$.

Table 2. Chemoselective reduction of 4-(2-nitrophenyl-, 3-nitrophenyl- and 4-nitrophenyl)- 2oxobut-3-enyl-1'-deoxy-glycopyranosides to quinolinemethyl- or aminophenyl 2-oxobut-3-enyl 1'-deoxy- glycopyranosides

\begin{tabular}{|c|c|c|c|c|}
\hline Entry & Substrate & $\begin{array}{c}\text { Reaction } \\
\text { time } \\
(\mathrm{min})\end{array}$ & Product & $\begin{array}{c}\text { Isolate } \\
\text { d yield } \\
(\%)\end{array}$ \\
\hline 1 & 1 & 110 & 5 & 60 \\
\hline 2 & $\mathrm{H}$ & 110 & & 66 \\
\hline 3 & $\mathrm{OH}$ & 105 & & 67 \\
\hline 4 & $3 \mathbf{a}$ & 100 & $7 \mathbf{a}$ & 63 \\
\hline 5 & $\begin{array}{rr}\text { OAc } & \| \\
& \text { O } \\
& 3 b\end{array}$ & 110 & & 67 \\
\hline 6 & $\begin{array}{r}\text { OAc }{ }_{\mathrm{O}} \\
\mathbf{3 c}\end{array}$ & 95 & $\mathrm{Ac}$ & 69 \\
\hline
\end{tabular}

Table 2 (continued) 

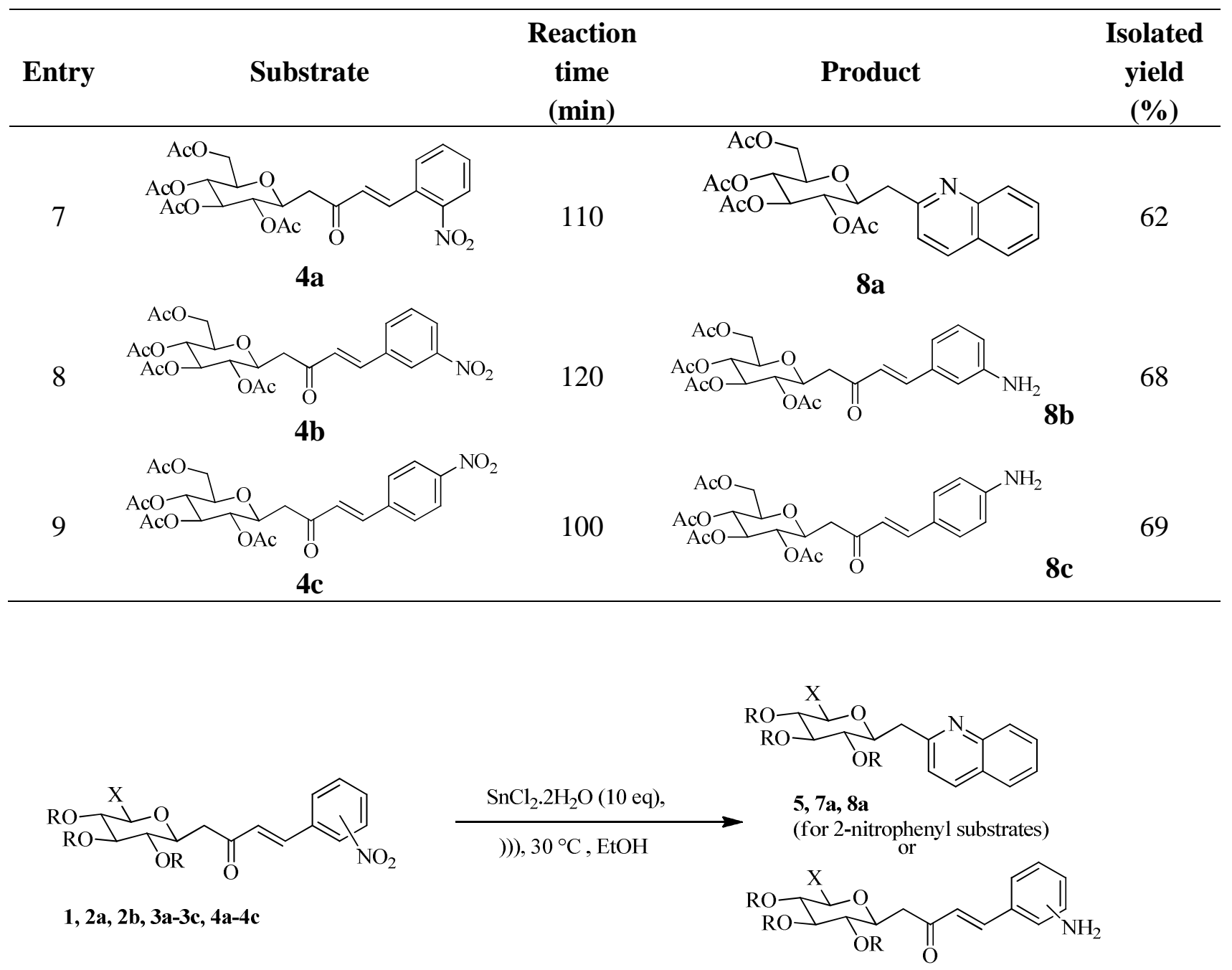

$6 a, 6 b, 7 b, 7 c, 8 b, 8 c$

(for 3,and 4-nitrophenyl substrates)

Scheme 3. Chemoselective reduction of nitrophenyl 2-oxobut-3-enyl-1'-deoxy-glycopyranosides to quinolinemethyl or aminophenyl 2-oxobut-3-enyl-1'-deoxy-glycopyranosides.

Further, to enhance the scope of the chemoselective reduction of the nitro group in such compounds with other sensitive functional groups in sugar moiety, we selected 6'-tosyloxy-1'deoxy-glucopyranoside derivative (9) and 6'-azido-1',6'-dideoxy-glucopyranoside (10) respectively. The latter could be prepared by selective tosylation of $(E)-4$-(3-nitrophenyl)-1-[1'deoxy- $\beta$-D-glucopyranos-1'-yl]but-3-en-2-one (2a) with $p$-toluenesulfonyl chloride to give the respective 6'-tosyloxy derivative (9), which on treatment with $\mathrm{NaN}_{3}$ in DMF gave 6'-azido-6deoxy derivative (10). The reduction of nitro groups in the above compounds 9 and $\mathbf{1 0}$ with $\mathrm{SnCl}_{2} \cdot 2 \mathrm{H}_{2} \mathrm{O}$ in ultrasonic bath as mentioned above resulted in respective 4-(aminophenyl)- 2- 
oxobut-3-enyl glycosides 11 and 12 respectively in good yields (Scheme 4). The structures of these two products were also established on the basis of their spectroscopic data.

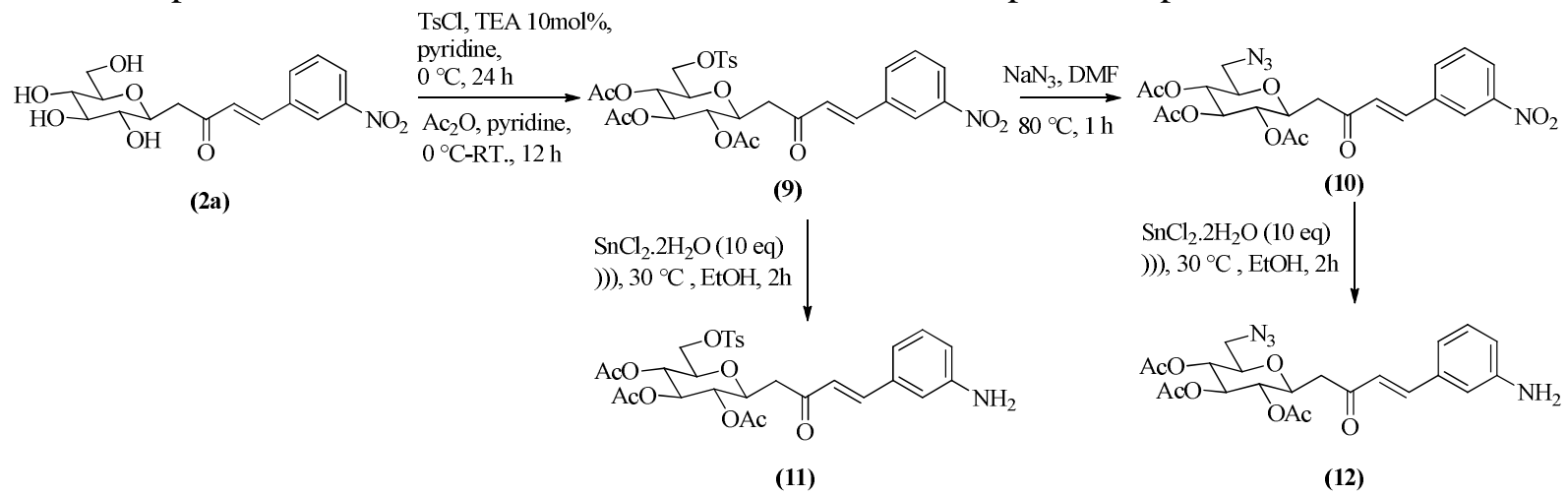

Scheme 4. Chemoselective reduction of nitrophenyl 2-oxobut-3-enyl-1'-deoxy-glycopyranosides with tosyl and azide functionalities.

The potential of these aminophenyl 2-oxobut-3-enyl-1'-deoxy-glycopyranosides as intermediates for the synthesis of various biologically important glycoconjugates have been demonstrated by selecting (E)-4-(3-aminophenyl)-1-[1'-deoxy-2',3',4',6'-tetra- $O$-acetyl- $\beta$-Dglucopyranos-1'-yl]-but-3-en-2-one (8b) for three different reactions as shown in scheme 5. Its reaction with $p$-toluenesulfonyl chloride in presence of $\mathrm{Et}_{3} \mathrm{~N}$ in $\mathrm{CH}_{2} \mathrm{Cl}_{2}$ at $0{ }^{\circ} \mathrm{C}$ to $\mathrm{RT}$ led to the formation of respective 4-[3-( $N$-sulfonylamino)phenyl]- 2-oxobut-3-enyl glycopyranoside (13) in good yield. Similarly reaction of $\mathbf{8 b}$ with phthalic anhydride and phenylisocyanate separately led to the formation of respective phenyl carbamoyl benzoic acid (14) and urea derivative (15) in very good yields. The structures of the isolated compounds were established on the basis of their spectroscopic data.

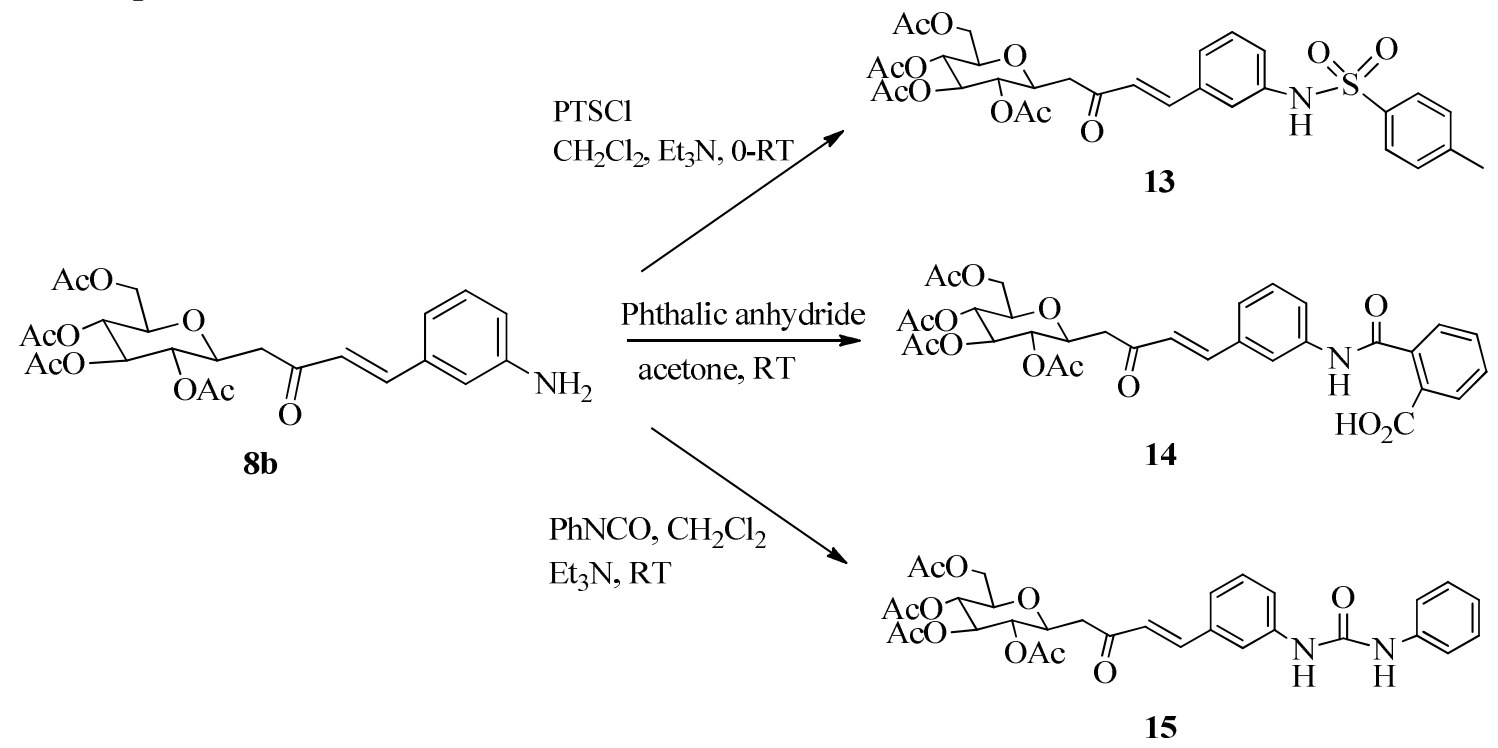


Scheme 5. Demonstrative examples of application of aminophenyl 2-oxobut-3-enyl-1'deoxyglycopyranoside for library generation.

\section{Conclusions}

We have prepared a series of 4-(nitrophenyl)- 2-oxobut-3-enyl-1'-deoxy-glycopyranosides from $\beta$ - $C$-glycosylic propanones derived from D-xylose and D-glucose. The 4-(nitrophenyl)- 2oxobut-3-enyl-1'-deoxy-glycopyranosides on chemoselective reduction with $\mathrm{SnCl}_{2} .2 \mathrm{H}_{2} \mathrm{O}$ in ultrasonic bath at ambient temparature resulted in the respective (E)-1-[(1'-deoxy- $\beta$-D(glycopyranos-1'-yl)]-4-(aminophenyl)-but-3-en-2-ones and quinolin-2-methyl- $C$ glycopyranosides stereoselectively in good yields. The potential of these synthesized aminophenyl 2-oxobut-3-enyl glycopyranosides has been demonstrated in diversity-oriented synthesis of a library of potentially biologically active glycoconjugates with sulfonamide, phenylcarbamoylbenzoic acid and ureide moieties.

\section{Experimental Section}

General. Commercially available reagent grade chemicals were used as received. All reactions were followed by TLC on Merck Kieselgel 60 F254, with detection by UV light, spraying $20 \%$ aq. $\mathrm{KMnO}_{4}$ solution and/or spraying $4 \%$ ethanolic $\mathrm{H}_{2} \mathrm{SO}_{4}$. Column chromatography was performed on Silica Gel (60-120 mesh, E. Merck). IR spectra were recorded as thin films or in $\mathrm{KBr}$ solution with a Perkin-Elmer Spectrum RX-1 (4000-450 $\left.\mathrm{cm}^{-1}\right)$ spectrophotometer. ${ }^{1} \mathrm{H}$ and ${ }^{13} \mathrm{C}$ NMR spectra were recorded on Bruker DRX $400 \mathrm{MHz}, 300 \mathrm{MHz}, 75 \mathrm{MHz}$ and $100 \mathrm{MHz}$ instruments, respectively, in $\mathrm{CDCl}_{3}$ and DMSO- $\mathrm{d}_{6}$. Chemical shift values are reported in ppm relative to TMS (tetramethylsilane) as the internal reference, unless otherwise stated; s (singlet), d (doublet), t (triplet), dd (double doublet), m (multiplet); $J$ in Hertz. HRMS were performed using a Quattro II (Micromass) instrument. Optical rotations were measured in a 1.0-dm tube with a Rudolf Autopol III polarimeter in $\mathrm{CHCl}_{3}$ and $\mathrm{MeOH}$. "RT" denotes room temperature.

General procedure for the preparation of $(E)-4-(2-n i t r o p h e n y l)-1-\left[1^{\prime}\right.$-deoxy- $\beta$-Dxylopyranos-1'-yl]but-3-en-2-one (1). To a stirring solution of 1-(1'-deoxy- $\beta$-D-xylopyranos-1'yl)-propan-2-one $\mathrm{e}^{37,38}(2.0 \mathrm{~g}, 10.52 \mathrm{mmol})$ and 2-nitrobenzaldehyde (1.9 g, $\left.12.63 \mathrm{mmol}\right)$ in $\mathrm{MeOH}(15.0 \mathrm{~mL})$, L-proline $(20 \mathrm{~mol} \%)$ and $\mathrm{Et}_{3} \mathrm{~N}(20 \mathrm{~mol} \%)$ was added and stirring continued at ambient temperature till the disappearance (TLC) of sugar ketone. The solvent was evaporated under reduced pressure to give a crude mass, which was purified by column ( $\mathrm{SiO}_{2}, 60-120$ mesh) chromatography using a gradient of $\mathrm{MeOH} / \mathrm{CHCl}_{3}$ as eluent to give the compound 1 as a white solid. Yield 57\%, $0.969 \mathrm{~g}, \mathrm{mp} 109-110{ }^{\circ} \mathrm{C} ; R_{\mathrm{f}} 0.6\left(8: 2, \mathrm{CHCl}_{3}-\mathrm{MeOH}\right) ;[\alpha]_{\mathrm{D}}{ }^{25}-0.65$ (c 0.1 , $\mathrm{MeOH})$; IR $\left(v_{\max }, \mathrm{cm}^{-1}\right): 3393,1642,1528,1352(\mathrm{~N}-\mathrm{O}), 1093,733 .{ }^{1} \mathrm{H}$ NMR (300 MHz , DMSO$\left.d_{6}+\mathrm{CDCl}_{3}\right): \delta_{\mathrm{H}} 2.50(2 \mathrm{H}, \mathrm{m}, \mathrm{H}-1), 2.75\left(1 \mathrm{H}, \mathrm{m}, \mathrm{H}-2^{\prime}\right), 2.99\left(1 \mathrm{H}, \mathrm{m}, \mathrm{H}-4^{\prime}\right), 3.03\left(1 \mathrm{H}, \mathrm{m}, \mathrm{H}-3^{\prime}\right)$, $3.22(3 \mathrm{H}, \mathrm{m},-\mathrm{OH}), 3.29$ (1H, m, H-5'b), 3.34 (1H, m, H-5'a ), $3.70\left(1 \mathrm{H}, \mathrm{m}, \mathrm{H}-1^{\prime}\right), 7.43(1 \mathrm{H}, \mathrm{m}$, 
$\mathrm{H}-3), 7.65(2 \mathrm{H}, \mathrm{m}, \mathrm{ArH}), 7.83(3 \mathrm{H}, \mathrm{m}, \mathrm{H}-4, \mathrm{ArH}) .{ }^{13} \mathrm{C} \mathrm{NMR}\left(50 \mathrm{MHz}, \mathrm{DMSO}-d_{6}+\mathrm{CDCl}_{3}\right): \delta_{\mathrm{C}}$ 45.6 (C-1), 64.1 (C-5'), $69.3\left(\mathrm{C}^{-4} 4^{\prime}\right), 69.6$ (C-3'), 73.1 (C-2'), 75.9 (C-1'), 121.0 (Ar-C), 123.4 (C3), 127.5 (Ar-C), 128.1 (Ar-C), 131.5 (Ar-C), 133.0 (Ar-C), 133.0 (Ar-C), 140.1 (C-4), 146.7 (Ar-C), $206.1(\mathrm{C}=\mathrm{O})$; HRMS: Calcd. Accurate mass for $\left(\mathrm{C}_{15} \mathrm{H}_{17} \mathrm{NNaO}_{7}\right)$ : 346.0903. Found $346.0911[\mathrm{M}+\mathrm{Na}]^{+}$.

(E)-4-(3-Nitrophenyl)-1-[1'-deoxy- $\beta$-D-glucopyranos-1'-yl]but-3-en-2-one $\quad(2 a) . \quad$ It $\quad$ was obtained by the reaction of 1-(1'-deoxy- $\beta$-D-glucopyranos-1'-yl)-propan-2-one ${ }^{37,38}$ (2.0 g, 9.09 mmol) and 3-nitrobenzaldehyde (1.64 g, $10.9 \mathrm{mmol})$ as a white solid, yield 68\%, $1.5 \mathrm{~g}$, mp 118$119{ }^{\circ} \mathrm{C} ; R_{\mathrm{f}} 0.6\left(8: 2, \mathrm{CHCl}_{3}-\mathrm{MeOH}\right) ;[\alpha]_{\mathrm{D}}{ }^{25}-0.61(\mathrm{c} 0.1, \mathrm{MeOH}) ; \mathrm{IR}\left(v_{\max }, \mathrm{cm}^{-1}\right): 3422,1640$, 1529, $1352(\mathrm{~N}-\mathrm{O}), 1088,681 .{ }^{1} \mathrm{H}$ NMR $\left(300 \mathrm{MHz}, \mathrm{DMSO}-d_{6}+\mathrm{CDCl}_{3}\right): \delta_{\mathrm{H}} 2.88(2 \mathrm{H}, \mathrm{m}, \mathrm{H}-1)$, 3.14 (1H, m, H-4'), 3.21 (2H, m, H-3', H-2'), 3.40 (3H, m, $3 \times-\mathrm{OH}), 3.65$ (4H, m, H-6', H-5', $\mathrm{OH}), 3.74\left(1 \mathrm{H}, \mathrm{m}, \mathrm{H}-1^{\prime}\right), 6.99(1 \mathrm{H}, \mathrm{d}, J .16 .2 \mathrm{~Hz}, \mathrm{H}-3), 7.64(2 \mathrm{H}, \mathrm{m}, \mathrm{H}-4, \mathrm{Ar}-\mathrm{H}), 7.96$ (1H, d, J.7.4 Hz, Ar-H), $8.21\left(1 \mathrm{H}, \mathrm{d}\right.$, J.5.9 Hz, Ar-H), $8.42(1 \mathrm{H}, \mathrm{s}, \mathrm{Ar}-\mathrm{H}) .{ }^{13} \mathrm{C}$ NMR (50 MHz , DMSO$\left.d_{6}+\mathrm{CDCl}_{3}\right): \delta_{\mathrm{C}} 43.9(\mathrm{C}-1) ; 62.0\left(\mathrm{C}-6^{\prime}\right), 70.7\left(\mathrm{C}-2^{\prime}\right), 73.8\left(\mathrm{C}-3^{\prime}\right), 76.0\left(\mathrm{C}-4^{\prime}\right), 77.4\left(\mathrm{C}-1^{\prime}\right), 77.9$ (C-5'), 122.6 (Ar-C), 124.3 (Ar-C), 129.2 (C-3), 134.0 (Ar-C), 136.6 (Ar-C), 139.6 (C-4), 148.6 (Ar-C), $197.9(\mathrm{C}=\mathrm{O})$; HRMS: Calcd. Accurate mass for $\left(\mathrm{C}_{16} \mathrm{H}_{19} \mathrm{NNaO}_{8}\right)$ : 376.1008. Found $376.0989[\mathrm{M}+\mathrm{Na}]^{+}$.

(E)-4-(4-Nitrophenyl)-1-[1'-deoxy- $\beta$-D-glucopyranos-1'-yl]but-3-en-2-one $\quad(2 \mathrm{~b}) . \quad$ It $\quad$ was obtained by the reaction of 1-(1'-deoxy- $\beta$-D-glucopyranos-1'-yl)-propan-2-one ${ }^{37,38}$ (2.0 g, 9.09 mmol) and 4-nitrobenzaldehyde (1.64 g, $10.9 \mathrm{mmol})$ as a white solid, yield 69\%, $1.52 \mathrm{~g}$, mp 123$124{ }^{\circ} \mathrm{C} ; R_{\mathrm{f}} 0.6\left(8: 2, \mathrm{CHCl}_{3}-\mathrm{MeOH}\right) ;[\alpha]_{\mathrm{D}}{ }^{25}-86\left(\mathrm{c} 0.1, \mathrm{CHCl}_{3}\right) ; \mathrm{IR}\left(v_{\max }, \mathrm{cm}^{-1}\right): 3402,1617,1520$, $1347(\mathrm{~N}-\mathrm{O}), 1088,749 .{ }^{1} \mathrm{H}$ NMR $\left(300 \mathrm{MHz}, \mathrm{DMSO}-d_{6}+\mathrm{CDCl}_{3}\right): \delta_{\mathrm{H}} 2.80(1 \mathrm{H}, \mathrm{m}, \mathrm{H}-1 \mathrm{~b}), 2.93$ (2H, m, H-5', H-1a), 3.01 (2H, m, H-3', H-4'), 3.04 (1H, m, -OH), $3.14(2 \mathrm{H}, \mathrm{m}, 2 \times-\mathrm{OH}), 3.21$ $(1 \mathrm{H}, \mathrm{m},-\mathrm{OH}), 3.65\left(2 \mathrm{H}, \mathrm{m},-\mathrm{OCH}_{2}\right), 3.76\left(2 \mathrm{H}, \mathrm{m}, \mathrm{H}-2^{\prime}, \mathrm{H}-6^{\prime}\right), 6.98(1 \mathrm{H}, \mathrm{d}, \mathrm{J} .16 .2 \mathrm{~Hz}, \mathrm{H}-3), 7.60$ $(1 \mathrm{H}, \mathrm{d}, J .16 .3 \mathrm{~Hz}, \mathrm{H}-4), 7.86(2 \mathrm{H}, \mathrm{d}, J .8 .4 \mathrm{~Hz}, \mathrm{Ar}-\mathrm{H}), 8.20(2 \mathrm{H}, \mathrm{d}, J .8 .5 \mathrm{~Hz}, \mathrm{Ar}-\mathrm{H}) .{ }^{13} \mathrm{C}$ NMR $(50$ $\left.\mathrm{MHz}, \mathrm{DMSO}-d_{6}+\mathrm{CDCl}_{3}\right): \delta_{\mathrm{C}} 43.9(\mathrm{C}-1), 56.9\left(-\mathrm{CH}_{2} \mathrm{OH}\right), 70.2\left(\mathrm{C}-3^{\prime}\right), 73.7\left(\mathrm{C}-4^{\prime}\right), 73.9\left(\mathrm{C}-5^{\prime}\right)$, 77.2 (C-2'), 78.9 (C-6'), 124.1 (Ar-C), 127.9 (C-3), 130.5 (Ar-C), 141.3 (C-4), 148.3 (Ar-C), $198.3(\mathrm{C}=\mathrm{O})$; HRMS: Calcd. Accurate mass for $\left(\mathrm{C}_{16} \mathrm{H}_{19} \mathrm{NNaO}_{8}\right)$ : 376.1008. Found 376.0994 $[\mathrm{M}+\mathrm{Na}]^{+}$.

General procedure for the preparation of $(E)-4$-(2-nitrophenyl)-1-[1'-deoxy-2',3', $4^{\prime}$-tri-Oacetyl- $\beta$-D-xylopyranos-1'-yl]but-3-en-2-one (3a). To a stirring solution of 1-(1'-deoxy-2',3',4'tri- $O$-acetyl- $\beta$-D-xylopyranos-1'-yl)-propan-2-one ${ }^{37,38} \quad(2.0 \quad \mathrm{~g}, \quad 6.32 \quad \mathrm{mmol}) \quad$ and 2nitrobenzaldehyde $(1.14 \mathrm{~g}, 7.59 \mathrm{mmol})$ in $\mathrm{CH}_{2} \mathrm{Cl}_{2}(15.0 \mathrm{~mL})$, pyrrolidine $(20 \mathrm{~mol} \%)$ was added and stirring continued at ambient temperature till the disappearance (TLC) of sugar ketone. The reaction mixture was extracted with $\mathrm{CH}_{2} \mathrm{Cl}_{2}$ and washed with water, the organic layer was dried (anhyd. $\mathrm{Na}_{2} \mathrm{SO}_{4}$ ) and solvent evaporated under reduced pressure to give a crude mass, which was purified by column $\left(\mathrm{SiO}_{2}, 60-120\right.$ mesh) chromatography using a gradient of EtOAc/Hexane as eluent to give the title compound $3 \mathrm{a}$ as a white solid, yield $65 \%, 1.84 \mathrm{~g}, \mathrm{mp} 116-117{ }^{\circ} \mathrm{C} ; R_{\mathrm{f}} 0.5$ $\left(6: 4\right.$, Hexane-EtOAc); $[\alpha]_{\mathrm{D}}{ }^{25}-64\left(\mathrm{c} 0.1, \mathrm{CHCl}_{3}\right) ; \mathrm{IR}\left(v_{\max }, \mathrm{cm}^{-1}\right): 3564,1638,1371(\mathrm{~N}-\mathrm{O}), 1220$, 
771. ${ }^{1} \mathrm{H}$ NMR $\left(300 \mathrm{MHz}, \mathrm{CDCl}_{3}+\mathrm{CCl}_{4}\right): \delta_{\mathrm{H}} 2.03\left(9 \mathrm{H}, \mathrm{m}, 3 \times-\mathrm{COCH}_{3}\right), 2.73\left(1 \mathrm{H}, \mathrm{dd}, J_{1} .2 .4 \mathrm{~Hz}\right.$, $\left.J_{2} .15 .9 \mathrm{~Hz}, \mathrm{H}-1 \mathrm{~b}\right), 3.02\left(1 \mathrm{H}, \mathrm{dd}, J_{1} .8 .3 \mathrm{~Hz}, J_{2} .15 .9 \mathrm{~Hz}, \mathrm{H}-1 \mathrm{a}\right), 3.36\left(1 \mathrm{H}, \mathrm{m}, \mathrm{H}-1^{\prime}\right), 4.09$ (2H, m, H-5'), 5.01 (2H, m, H-2', H-4'), 5.22 (1H, t, J.9.3 Hz, H-3'), 6.61 (1H, d, J.16.0 Hz, H-3), 7.56 (1H, m, Ar-H), 7.64 (2H, m, Ar-H), $8.01(1 \mathrm{H}, \mathrm{d}, J .16 .0 \mathrm{~Hz}, \mathrm{H}-4), 8.08$ (1H, d, J.7.92 Hz, Ar-H). ${ }^{13} \mathrm{C}$ NMR $\left(100 \mathrm{MHz}, \mathrm{CDCl}_{3}+\mathrm{CCl}_{4}\right): \delta_{\mathrm{C}} 20.6\left(3 \times-\mathrm{COCH}_{3}\right), 42.6(\mathrm{C}-1), 66.7\left(\mathrm{C}-5^{\prime}\right), 69.2\left(\mathrm{C}-4^{\prime}\right)$, 71.6 (C-3'), $71.9\left(\mathrm{C}-2^{\prime}\right), 73.7$ (C-1'), 125.0 (Ar-C), 129.0 (C-3), 130.4 (Ar-C), 130.8 (Ar-C), 131.2 (Ar-C), 133.4 (Ar-C), 139.0 (C-4), 148.4 (Ar-C), 169.4, 169.6, 169.9 (3 × - $\left.\mathrm{COCH}_{3}\right), 195.6$ $(\mathrm{C}=\mathrm{O})$; HRMS: Calcd. Accurate mass for $\left(\mathrm{C}_{21} \mathrm{H}_{23} \mathrm{NNaO}_{10}\right)$ : 472.1220. Found 472.1229 $[\mathrm{M}+\mathrm{Na}]^{+}$.

(E)-4-(3-Nitrophenyl)-1-[1'-deoxy-2',3',4'-tri-O-acetyl- $\beta$-D-xylopyranos-1'-yl]but-3-en-2-one (3b): It was obtained by the reaction of 1 -(1'-deoxy-2',3',4'-tri- $O$-acetyl- $\beta$-D-xylopyranos- $1^{\prime}$-yl)propan-2-one $^{37,38}(2.0 \mathrm{~g}, 6.32 \mathrm{mmol})$ and 3-nitrobenzaldehyde $(1.14 \mathrm{~g}, 7.59 \mathrm{mmol})$ as a white solid, yield 65\%, $1.98 \mathrm{~g}, \mathrm{mp} 109-112{ }^{\circ} \mathrm{C} ; R_{\mathrm{f}} 0.6\left(6: 4\right.$, Hexane-EtOAc); $[\alpha]_{\mathrm{D}}{ }^{25}-43$ (c 0.1, $\left.\mathrm{CHCl}_{3}\right)$; IR $\left(v_{\max }, \mathrm{cm}^{-1}\right): 3434,1751(\mathrm{C}=\mathrm{O}), 1619(\mathrm{C}=\mathrm{C}), 1533,1360(\mathrm{~N}-\mathrm{O}), 1227,732 .{ }^{1} \mathrm{H}$ NMR (300 $\left.\mathrm{MHz}, \mathrm{CDCl}_{3}+\mathrm{CCl}_{4}\right): \delta_{\mathrm{H}} 2.03\left(9 \mathrm{H}, \mathrm{m}, 3 \times-\mathrm{COCH}_{3}\right), 2.69\left(1 \mathrm{H}, \mathrm{dd}, J_{1} .2 .97 \mathrm{~Hz}, J_{2} .16 .0 \mathrm{~Hz}, \mathrm{H}-1 \mathrm{~b}\right)$, $3.02\left(1 \mathrm{H}, \mathrm{dd}, J_{1} .8 .6 \mathrm{~Hz}, J_{2} 16.0 \mathrm{~Hz}, \mathrm{H}-1 \mathrm{a}\right), 3.36\left(1 \mathrm{H}, \mathrm{m}, \mathrm{H}-1^{\prime}\right), 4.08\left(2 \mathrm{H}, \mathrm{m}, \mathrm{H}-5^{\prime}\right), 5.00-4.87$ (2H, m, H-2', H-4'), 5.23 (1H, t, J.9.3 Hz, H-3'), 6.87 (1H, d, J.16.1 Hz, H-3), 7.63-7.55 (2H, m, ArH, H-4), $7.86(1 \mathrm{H}, \mathrm{d}, J .7 .6 \mathrm{~Hz}, \mathrm{ArH}), 8.27(1 \mathrm{H}, \mathrm{d}, J .8 .0 \mathrm{~Hz}, \mathrm{ArH}), 8.41(1 \mathrm{H}, \mathrm{s}, \mathrm{ArH}) .{ }^{13} \mathrm{C} \mathrm{NMR}$ $\left(50 \mathrm{MHz}, \mathrm{CDCl}_{3}+\mathrm{CCl}_{4}\right): \delta_{\mathrm{C}} 20.6\left(3 \times-\mathrm{COCH}_{3}\right), 43.0(\mathrm{C}-1), 66.7\left(\mathrm{C}-5^{\prime}\right), 69.1\left(\mathrm{C}-2^{\prime}\right), 71.8\left(\mathrm{C}-4^{\prime}\right)$, 73.6 (C-3'), 74.7 (C-1'), 122.6 (Ar-C), 124.7 (Ar-C), 128.6 (C-3), 129.9 (Ar-C), 133.7 (Ar-C), 136.1 (Ar-C), 140.3 (C-4), 148.8 (Ar-C), 169.4, 169.8, 175.9 (3 $\left.\times-\mathrm{COCH}_{3}\right), 195.4(\mathrm{C}=\mathrm{O})$; ESIMS: $m / z 472.2(\mathrm{M}+\mathrm{Na})^{+}$; HRMS: Calcd. Accurate mass for $\left(\mathrm{C}_{21} \mathrm{H}_{23} \mathrm{NNaO}_{10}\right): 472.1220$. Found $472.1204[\mathrm{M}+\mathrm{Na}]^{+}$.

(E)-4-(4-Nitrophenyl)-1-[1'-deoxy-2',3',4'-tri-O-acetyl- $\beta$-D-xylopyranos-1'-yl]but-3-en-2-one (3c). It was obtained by the reaction of 1 -( $1^{\prime}$-deoxy-2',3',4'-tri- $O$-acetyl- $\beta$-D-xylopyranos- $1^{\prime}$-yl)propan-2-one $^{37,38}(2.0 \mathrm{~g}, 6.32 \mathrm{mmol})$ and 4-nitrobenzaldehyde $(1.14 \mathrm{~g}, 7.59 \mathrm{mmol})$ as a white solid, yield 71\%, $2.01 \mathrm{~g}$, mp 110-111 ${ }^{\circ} \mathrm{C} ; R_{\mathrm{f}} 0.6$ (6:4, Hexane-EtOAc); $[\alpha]_{\mathrm{D}}{ }^{25}-67\left(\mathrm{c} 0.1, \mathrm{CHCl}_{3}\right)$; IR $\left(v_{\max }, \mathrm{cm}^{-1}\right): 3447,1736(\mathrm{C}=\mathrm{O}), 1624(\mathrm{C}=\mathrm{C}), 1521,1347(\mathrm{~N}-\mathrm{O}), 1234,746 .{ }^{1} \mathrm{H}$ NMR (300 $\left.\mathrm{MHz}, \mathrm{CDCl}_{3}+\mathrm{CCl}_{4}\right): \delta_{\mathrm{H}} 2.02\left(9 \mathrm{H}, \mathrm{m}, 3 \times-\mathrm{COCH}_{3}\right), 2.68\left(1 \mathrm{H}, \mathrm{dd}, J_{1} .2 .5 \mathrm{~Hz}, J_{2} .16 .0 \mathrm{~Hz}, \mathrm{H}-1 \mathrm{~b}\right)$, $3.01\left(1 \mathrm{H}, \mathrm{dd}, J_{1} .8 .6 \mathrm{~Hz}, J_{2} .16 .0 \mathrm{~Hz}, \mathrm{H}-1 \mathrm{a}\right), 3.34\left(1 \mathrm{H}, \mathrm{m}, \mathrm{H}-1^{\prime}\right), 4.06$ (2H, m, H-5'), 4.98-4.85 $\left(2 \mathrm{H}, \mathrm{m}, \mathrm{H}-2^{\prime}, \mathrm{H}-4^{\prime}\right), 5.22$ (1H, t, J.9.3 Hz, H-3'), 6.85 (1H, d, J.16.1 Hz, H-3), 7.58 (1H, d, J.16.1 $\mathrm{Hz}, \mathrm{H}-4), 7.72(2 \mathrm{H}, \mathrm{d}, J .8 .5 \mathrm{~Hz}, \mathrm{ArH}), 8.27(2 \mathrm{H}, \mathrm{d}, J .8 .5 \mathrm{~Hz}, \mathrm{ArH}) .{ }^{13} \mathrm{C} \mathrm{NMR}(50 \mathrm{MHz}$, $\left.\mathrm{CDCl}_{3}+\mathrm{CCl}_{4}\right): \delta_{\mathrm{C}} 20.6\left(3 \times-\mathrm{COCH}_{3}\right), 42.9(\mathrm{C}-1), 66.7\left(\mathrm{C}-5^{\prime}\right), 69.1\left(\mathrm{C}-2^{\prime}\right), 71.8\left(\mathrm{C}-4^{\prime}\right), 73.5(\mathrm{C}-$ 3'), 74.6 (C-1'), 124.1 (Ar-C), 128.8 (C-3), 129.5 (Ar-C), 130.3 (Ar-C), 140.2 (C-4), 148.7 (ArC), 169.4, 169.7, $169.8\left(3 \times-\mathrm{COCH}_{3}\right), 195.4(\mathrm{C}=\mathrm{O})$; HRMS: Calcd. Accurate mass for $\left(\mathrm{C}_{21} \mathrm{H}_{23} \mathrm{NNaO}_{10}\right)$ : 472.1220. Found 472.1205 [M+Na] ${ }^{+}$.

(E)-4-(2-Nitrophenyl)-1-[1'-deoxy-2',3',4',6'-tetra- $O$-acetyl- $\beta$-D-glucopyranos-1'-yl]but-3en-2-one (4a). It was obtained by the reaction of 1 -( $1^{\prime}$-deoxy-2', $3^{\prime}, 4^{\prime}, 6^{\prime}$-tetra- $O$-acetyl- $\beta$-Dglucopyranos-1'-yl)-propan-2-one ${ }^{37,38}(2.0 \mathrm{~g}, 5.15 \mathrm{mmol})$ and 2-nitrobenzaldehyde $(0.93 \mathrm{~g}, 6.18$ $\mathrm{mmol})$ as a white solid, yield $67 \%, 1.79 \mathrm{~g}, \mathrm{mp} 104-105{ }^{\circ} \mathrm{C} ; R_{\mathrm{f}} 0.5\left(5: 5\right.$, Hexane-EtOAc); $[\alpha]_{\mathrm{D}}{ }^{25}$ - 
$18\left(\mathrm{c} 0.1, \mathrm{CHCl}_{3}\right)$; IR $\left(v_{\max }, \mathrm{cm}^{-1}\right): 3023,1749(\mathrm{C}=\mathrm{O}), 1615(\mathrm{C}=\mathrm{C}), 1527,1370(\mathrm{~N}-\mathrm{O}), 1224,763$. ${ }^{1} \mathrm{H}$ NMR $\left(300 \mathrm{MHz}, \mathrm{CDCl}_{3}+\mathrm{CCl}_{4}\right): \delta_{\mathrm{H}} 2.04\left(12 \mathrm{H}, \mathrm{m}, 4 \times-\mathrm{COCH}_{3}\right), 2.74\left(1 \mathrm{H}, \mathrm{dd}, J_{1} .3 .0 \mathrm{~Hz}\right.$, $\left.J_{2} .16 .3 \mathrm{~Hz}, \mathrm{H}-1 \mathrm{~b}\right), 3.05\left(1 \mathrm{H}, \mathrm{dd}, J_{1} .8 .2 \mathrm{~Hz}, J_{2} .16 .2 \mathrm{~Hz}, \mathrm{H}-1 \mathrm{a}\right), 3.71\left(1 \mathrm{H}, \mathrm{m}, \mathrm{H}-1^{\prime}\right), 4.10$ (2H, m, H-6'), 4.25 (1H, m, H-5'), $4.96\left(1 \mathrm{H}, \mathrm{t}, J .9 .6 \mathrm{~Hz}, \mathrm{H}-2^{\prime}\right), 5.06\left(1 \mathrm{H}, \mathrm{t}, J .9 .7 \mathrm{~Hz}, \mathrm{H}-4^{\prime}\right), 5.21$ (1H, t, J.9.3 Hz, H-3'), 6.60 (1H, d, J.16.0 Hz, H-3), 7.58 (1H, m, Ar-H), 7.63 (2H, m, Ar-H), 7.99 (1H, d, J.16.0 Hz, H-4), $8.06(1 \mathrm{H}, \mathrm{d}, J .7 .9 \mathrm{~Hz}, \mathrm{Ar}-\mathrm{H}) .{ }^{13} \mathrm{C} \mathrm{NMR}\left(75 \mathrm{MHz}, \mathrm{CDCl}_{3}+\mathrm{CCl}_{4}\right): \delta_{\mathrm{C}} 20.5(4 \times$ $\left.-\mathrm{COCH}_{3}\right), 42.6(\mathrm{C}-1), 61.8\left(\mathrm{C}-6^{\prime}\right), 68.3\left(\mathrm{C}-2^{\prime}\right), 71.6\left(\mathrm{C}-4^{\prime}\right), 74.0\left(\mathrm{C}-3^{\prime}\right), 74.1\left(\mathrm{C}-1^{\prime}\right), 75.7\left(\mathrm{C}-5^{\prime}\right)$, 125.0 (Ar-C), 129.0 (C-3), 130.4 (Ar-C), 130.63 (Ar-C), 130.66 (Ar-C), 133.4 (Ar-C), 138.9 (C4), 148.4 (Ar-C), 169.2, 169.6, 169.9, 170.2 (4 × - $\left.\mathrm{COCH}_{3}\right), 195.4(\mathrm{C}=\mathrm{O})$; HRMS: Calcd. Accurate mass for $\left(\mathrm{C}_{24} \mathrm{H}_{27} \mathrm{NNaO}_{12}\right): 544.1431$. Found $544.1419[\mathrm{M}+\mathrm{Na}]^{+}$.

(E)-4-(3-Nitrophenyl)-1-[1'-deoxy-2',3',4',6'-tetra- $O$-acetyl- $\beta$-D-glucopyranos-1'-yl]but-3-

en-2-one (4b). It was obtained by the reaction of 1 -( $1^{\prime}$-deoxy-2',3',4',6'-tetra- $O$-acetyl- $\beta$-Dglucopyranos-1'-yl)-propan-2-one ${ }^{37,38}(2.0 \mathrm{~g}, 5.15 \mathrm{mmol})$ and 3-nitrobenzaldehyde $(0.93 \mathrm{~g}, 6.18$ mmol) as a white solid, yield $70 \%, 1.87 \mathrm{~g}, \mathrm{mp} 73-75^{\circ} \mathrm{C} ; R_{\mathrm{f}} 0.5\left(5: 5\right.$, Hexane-EtOAc); $[\alpha]_{\mathrm{D}}{ }^{25}-25$ (c 0.1, $\left.\mathrm{CHCl}_{3}\right)$; IR $\left(v_{\max }, \mathrm{cm}^{-1}\right):$ 3074, $1747(\mathrm{C}=\mathrm{O}), 1668(\mathrm{C}=\mathrm{C}), 1535,1365(\mathrm{~N}-\mathrm{O}), 1225,736$. ${ }^{1} \mathrm{H}$ NMR $\left(300 \mathrm{MHz}, \mathrm{CDCl}_{3}+\mathrm{CCl}_{4}\right): \delta_{\mathrm{H}} 2.03\left(12 \mathrm{H}, \mathrm{m}, 4 \times-\mathrm{COCH}_{3}\right), 2.72(1 \mathrm{H}, \mathrm{m}, \mathrm{H}-1 \mathrm{~b}), 3.01$ (1H, m, H-1a), 3.72 (1H, m, H-1'), 4.10-4.00 (2H, m, H-6'), 4.27 (1H, m, H-5'), 4.99 (1H, t, J.9.6 Hz, H-2'), 5.09 (1H, t, J.9.7 Hz, H-4'), 5.21 (1H, t, J.9.3 Hz, H-3'), 6.87 (1H, d, J.16.1 Hz, H-3), $7.63(2 \mathrm{H}, \mathrm{m}, \mathrm{H}-4, \mathrm{Ar}-\mathrm{H}), 7.87(1 \mathrm{H}, \mathrm{d}, J .7 .7 \mathrm{~Hz}, \mathrm{Ar}-\mathrm{H}), 8.27$ (1H, d, J.8.1 Hz, Ar-H), $8.42(1 \mathrm{H}, \mathrm{s}$, $\mathrm{Ar}-\mathrm{H}) .{ }^{13} \mathrm{C}$ NMR $\left(75 \mathrm{MHz}, \mathrm{CDCl}_{3}+\mathrm{CCl}_{4}\right): \delta_{\mathrm{C}} 20.5\left(4 \times-\mathrm{COCH}_{3}\right), 43.0(\mathrm{C}-1), 61.7(\mathrm{C}-6$ '), 68.3 (C-2'), 74.0 (C-3'), 71.6 (C-4'), 75.8 (C-1'), 76.5 (C-5'), 122.6 (Ar-C), 124.7 (Ar-C), 128.6 (C-3), 129.9 (Ar-C), 133.6 (Ar-C), 136.1 (Ar-C), 140.2 (C-4), 148.8 (Ar-C), 169.0, 169.5, 169.7, 170.0 $\left(4 \times-\mathrm{COCH}_{3}\right), 195.0(\mathrm{C}=\mathrm{O})$; HRMS: Calcd. Accurate mass for $\left(\mathrm{C}_{24} \mathrm{H}_{27} \mathrm{NNaO}_{12}\right): 544.1431$. Found 544.1408 [M+Na $]^{+}$.

(E)-4-(4-Nitrophenyl)-1-[1'-deoxy-2',3',4',6'-tetra- $O$-acetyl- $\beta$-D-glucopyranos-1'-yl]but-3en-2-one (4c). It was obtained by the reaction of 1 -( $1^{\prime}$-deoxy-2',3',4',6'-tetra- $O$-acetyl- $\beta$-Dglucopyranos-1'-yl)-propan-2-one ${ }^{37,38}(2.0 \mathrm{~g}, 5.15 \mathrm{mmol})$ and 4-nitrobenzaldehyde $(0.93 \mathrm{~g}, 6.18$ $\mathrm{mmol})$ as a white solid, yield $72 \%, 1.92 \mathrm{~g}, \mathrm{mp} 121-122^{\circ} \mathrm{C} ; R_{\mathrm{f}} 0.5\left(5: 5\right.$, Hexane-EtOAc); $[\alpha]_{\mathrm{D}}{ }^{25}$ $17\left(\mathrm{c} \mathrm{0.1}, \mathrm{CHCl}_{3}\right)$; IR $\left(v_{\max }, \mathrm{cm}^{-1}\right): 3248,1749(\mathrm{C}=\mathrm{O}), 1611(\mathrm{C}=\mathrm{C}), 1522,1347(\mathrm{~N}-\mathrm{O}), 1226,694$. ${ }^{1} \mathrm{H}$ NMR $\left(300 \mathrm{MHz}, \mathrm{CDCl}_{3}+\mathrm{CCl}_{4}\right): \delta_{\mathrm{H}} 2.02\left(12 \mathrm{H}, \mathrm{m}, 4 \times-\mathrm{COCH}_{3}\right), 2.71\left(1 \mathrm{H}, \mathrm{dd}, J_{1} .3 .2 \mathrm{~Hz}\right.$, $\left.J_{2} .16 .2 \mathrm{~Hz}, \mathrm{H}-1 \mathrm{~b}\right), 3.06\left(1 \mathrm{H}, \mathrm{dd}, J_{1} .8 .5 \mathrm{~Hz}, J_{2} .16 .2 \mathrm{~Hz}, \mathrm{H}-1 \mathrm{a}\right), 3.72\left(1 \mathrm{H}, \mathrm{m}, \mathrm{H}-1^{\prime}\right), 4.12-4.00(2 \mathrm{H}$, m, H-6'), 4.28 (1H, m, H-5'), 4.99 (1H, t, J.9.7 Hz, H-2'), 5.08 (1H, t, J.9.7 Hz, H-4'), 5.24 (1H, t, J.9.2 Hz, H-3'), 6.86 (1H, d, J.16.1 Hz, H-3), 7.59 (1H, d, J.16.1 Hz, H-4), 7.73 (2H, d, J.8.3 Hz, $\mathrm{Ar}-\mathrm{H}), 8.28(2 \mathrm{H}, \mathrm{d}, J .8 .2 \mathrm{~Hz}, \mathrm{Ar}-\mathrm{H}) .{ }^{13} \mathrm{C} \mathrm{NMR}\left(75 \mathrm{MHz}, \mathrm{CDCl}_{3}+\mathrm{CCl}_{4}\right): \delta_{\mathrm{C}} 20.6\left(4 \times-\mathrm{COCH}_{3}\right)$, $43.0\left(\mathrm{C}-1^{\prime}\right), 61.7\left(\mathrm{C}^{\prime} 6^{\prime}\right), 68.3\left(\mathrm{C}-2^{\prime}\right), 71.6\left(\mathrm{C}-4^{\prime}\right), 74.0\left(\mathrm{C}-3^{\prime}\right), 75.8\left(\mathrm{C}-1^{\prime}\right), 76.5(\mathrm{C}-5), 124.2(\mathrm{Ar}-$ C), 128.8 (C-3), 129.5 (Ar-C), 140.2 (Ar-C), 140.3 (C-4), 148.7 (Ar-C), 169.2, 169.6, 169.8, $170.1\left(4 \times-\mathrm{COCH}_{3}\right), 195.2(\mathrm{C}=\mathrm{O})$; HRMS: Calcd. Accurate mass for $\left(\mathrm{C}_{24} \mathrm{H}_{27} \mathrm{NNaO}_{12}\right)$ : 544.1431. Found 544.1420 [M+Na $]^{+}$.

General procedure for chemoselective reduction of nitro group in nitrophenyl 2-oxobut-3enyl glycopyranosides. To a stirring ethanolic solution of 4-(nitrophenyl)-2-oxobut-3-enyl 
glycopyranosides (1.0 equiv.) in ultrasonic bath at $30{ }^{\circ} \mathrm{C}, \mathrm{SnCl}_{2} \cdot 2 \mathrm{H}_{2} \mathrm{O}$ (10.0 equiv.) was added and reaction continued till the completion of the reaction. The reaction mixture was taken out of the ultrasonic bath and was neutralized by solid $\mathrm{NaHCO}_{3}$ and was filtered with celite pad then the filtrate was evaporated under reduced pressure and extracted with EtOAc and water. The EtOAc layer was dried (anhyd. $\mathrm{Na}_{2} \mathrm{SO}_{4}$ ) and solvent evaporated under reduced pressure to give a crude mass. The latter was purified by column chromatography $\left(\mathrm{SiO}_{2}, 60-120\right.$ mesh) using appropriate eluent to give the respective compounds in 60-70\% yields.

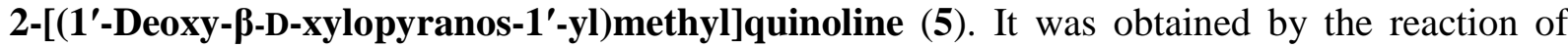
(2-nitrophenyl)- 2-oxobut-3-enyl glycopyranoside $\mathbf{1}$ (1.0 g, $3.09 \mathrm{mmol})$ with $\mathrm{SnCl}_{2} .2 \mathrm{H}_{2} \mathrm{O}(6.98$ $\mathrm{g}, 30.95 \mathrm{mmol})$ as a yellow solid, yield $60 \%, 0.51 \mathrm{~g}, \mathrm{mp} 132-133{ }^{\circ} \mathrm{C} ; R_{\mathrm{f}} 0.6\left(8: 2, \mathrm{CHCl}_{3}-\mathrm{MeOH}\right)$; $[\alpha]_{\mathrm{D}}{ }^{25}-33(\mathrm{c} 0.1, \mathrm{MeOH}) ; \mathrm{IR}\left(v_{\max }, \mathrm{cm}^{-1}\right): 3267,1216,1064,765 .{ }^{1} \mathrm{H}$ NMR (400 MHz, DMSO$\left.d_{6}\right): \delta_{\mathrm{H}} 3.07-2.9\left(4 \mathrm{H}, \mathrm{m}, \mathrm{H}-1, \mathrm{H}-2^{\prime}, \mathrm{H}^{-} 4^{\prime}\right), 3.17\left(1 \mathrm{H}, \mathrm{t}, J .8 .7 \mathrm{~Hz}, \mathrm{H}-3^{\prime}\right), 3.29\left(2 \mathrm{H}, \mathrm{m}, \mathrm{H}-5^{\prime}\right), 3.57$ $(1 \mathrm{H}, \mathrm{m},-\mathrm{OH}), 3.62(1 \mathrm{H}, \mathrm{m},-\mathrm{OH}), 3.64(1 \mathrm{H}, \mathrm{m},-\mathrm{OH}), 3.67\left(1 \mathrm{H}, \mathrm{m}, \mathrm{H}-1^{\prime}\right), 7.52(1 \mathrm{H}, \mathrm{d}, J .8 .6 \mathrm{~Hz}$, Ar-H), $7.71(1 \mathrm{H}, \mathrm{t}$, J.7.2 Hz, Ar-H), 7.83 (1H, t, J.7.3 Hz, Ar-H), 7.88 (1H, d, J.8.6 Hz, Ar-H), $8.06(1 \mathrm{H}, \mathrm{d}, J .8 .0 \mathrm{~Hz}, \mathrm{Ar}-\mathrm{H}), 8.58(1 \mathrm{H}, \mathrm{d}, J .8 .6 \mathrm{~Hz}, \mathrm{Ar}-\mathrm{H}) .{ }^{13} \mathrm{C}$ NMR $\left(50 \mathrm{MHz}, \mathrm{DMSO}-d_{6}\right.$ $\left.+\mathrm{CDCl}_{3}\right): \delta_{\mathrm{C}} 33.4\left(-\mathrm{CH}_{2^{-}}\right), 69.2\left(\mathrm{C}^{\prime} 5^{\prime}\right), 72.9\left(\mathrm{C}-4^{\prime}\right), 77.0\left(\mathrm{C}-3^{\prime}\right), 77.4\left(\mathrm{C}-2^{\prime}\right), 77.7\left(\mathrm{C}-1^{\prime}\right), 119.1$ (Ar-C), 123.9 (Ar-C), 125.4 (Ar-C), 127.6 (Ar-C), 127.7 (Ar-C), 128.8 (Ar-C), 129.9 (Ar-C), 140.5 (Ar-C), 145.9 (Ar-C); HRMS: Calcd. Accurate mass for $\left(\mathrm{C}_{15} \mathrm{H}_{17} \mathrm{NKO}_{4}\right)$ : 314.0795 . Found $314.1002[\mathrm{M}+\mathrm{K}]^{+}$.

(E)-4-(3-Aminophenyl)-1-[1'-deoxy- $\beta$-D-glucopyranos-1'-yl]but-3-en-2-one $\quad(6 a) . \quad$ It was obtained by the reaction of 3-nitrophenyl 2-oxobut-3-enyl glucopyranoside 2a (1.0 g, $2.83 \mathrm{mmol})$ with $\mathrm{SnCl}_{2} .2 \mathrm{H}_{2} \mathrm{O}(6.39 \mathrm{~g}, 28.3 \mathrm{mmol})$ as a yellow solid, yield $66 \%, 0.6 \mathrm{~g}, \mathrm{mp} 186-187{ }^{\circ} \mathrm{C} ; R_{\mathrm{f}} 0.5$ $\left(8: 2, \mathrm{CHCl}_{3}-\mathrm{MeOH}\right) ;[\alpha]_{\mathrm{D}}{ }^{25}-4(\mathrm{c} 0.1, \mathrm{MeOH}) ; \mathrm{IR}\left(v_{\max }, \mathrm{cm}^{-1}\right): 3394(\mathrm{~N}-\mathrm{H}), 1657(\mathrm{C}=\mathrm{C}), 1218$, 771. ${ }^{1} \mathrm{H}$ NMR (300 MHz, DMSO- $\left.d_{6}+\mathrm{CDCl}_{3}\right): \delta_{\mathrm{H}} 2.50(1 \mathrm{H}, \mathrm{m}, \mathrm{H}-1 \mathrm{a}), 2.77(1 \mathrm{H}, \mathrm{m}, \mathrm{H}-1 \mathrm{~b}), 2.98$ $\left(2 \mathrm{H}, \mathrm{m}, \mathrm{H}-2^{\prime}, \mathrm{H}-4^{\prime}\right), 3.13\left(3 \mathrm{H}, \mathrm{m}, \mathrm{H}-3^{\prime}, 2 \times-\mathrm{OH}\right), 3.19(2 \mathrm{H}, \mathrm{m}, 2 \times-\mathrm{OH}), 3.81\left(4 \mathrm{H}, \mathrm{m}, \mathrm{H}-1^{\prime}, \mathrm{H}-\right.$ 6', H-5'), 4.93 (2H, m, -NH $), 6.68(1 \mathrm{H}, \mathrm{d}, J .9 .1 \mathrm{~Hz}, \mathrm{Ar}-\mathrm{H}), 6.73(1 \mathrm{H}, \mathrm{s}, \mathrm{Ar}-\mathrm{H}), 6.85$ (2H, m, H-3, Ar-H), 7.06 (1H, t, J.7.3 Hz, Ar-H), $7.41(1 \mathrm{H}, \mathrm{d}, J .15 .9 \mathrm{~Hz}, \mathrm{H}-4) .{ }^{13} \mathrm{C}$ NMR (50 MHz, DMSO$\left.d_{6}+\mathrm{CDCl}_{3}\right): \delta_{\mathrm{C}} 43.3(\mathrm{C}-1), 62.5\left(\mathrm{C}-6^{\prime}\right), 70.1\left(\mathrm{C}-2^{\prime}\right), 73.4\left(\mathrm{C}-3^{\prime}\right), 75.7\left(\mathrm{C}-4^{\prime}\right), 77.9\left(\mathrm{C}-1^{\prime}\right), 78.5(\mathrm{C}-$ 5'), 119.3 (Ar-C), 122.6 (Ar-C), 124.1 (C-3), 127.2 (Ar-C), 129.7 (Ar-C), 135.6 (Ar-C), 141.2 (C-4), 151.0 (Ar-C), 197.8 (C=O); HRMS: Calcd. Accurate mass for $\left(\mathrm{C}_{16} \mathrm{H}_{21} \mathrm{NNaO}_{6}\right)$ : 346.1267 . Found $346.1254[\mathrm{M}+\mathrm{Na}]^{+}$.

(E)-4-(4-Aminophenyl)-1-[1'-deoxy- $\beta$-D-glucopyranos-1'-yl]but-3-en-2-one $\quad(6 \mathrm{~b}) . \quad$ It $\quad$ was obtained by the reaction of 4-nitrophenyl 2-oxobut-3-enyl glucopyranoside $2 \mathbf{b}(1.0 \mathrm{~g}, 2.83$ mmol) with $\mathrm{SnCl}_{2} .2 \mathrm{H}_{2} \mathrm{O}(6.39 \mathrm{~g}, 28.3 \mathrm{mmol})$ as a light yellow solid, yield 67\%, $0.6 \mathrm{~g}$, mp 185$187{ }^{\circ} \mathrm{C} ; R_{\mathrm{f}} 0.5\left(8: 2, \mathrm{CHCl}_{3}-\mathrm{MeOH}\right) ;[\alpha]_{\mathrm{D}}{ }^{25}-16(\mathrm{c} 0.1, \mathrm{MeOH}) ; \mathrm{IR}\left(v_{\max }, \mathrm{cm}^{-1}\right): 3355(\mathrm{~N}-\mathrm{H})$, 1661, 1226, 770. ${ }^{1} \mathrm{H}$ NMR $\left(300 \mathrm{MHz}, \mathrm{DMSO}-d_{6}+\mathrm{CDCl}_{3}\right): \delta_{\mathrm{H}} 2.59(2 \mathrm{H}, \mathrm{m}, \mathrm{H}-1), 2.83(1 \mathrm{H}, \mathrm{m}, \mathrm{H}-$ $\left.1^{\prime}\right), 3.03\left(1 \mathrm{H}, \mathrm{m}, \mathrm{H}-5^{\prime}\right), 3.17$ (2H, m, H-2', H-4'), 3.26 (1H, t, J.4.3 Hz, H-3'), 3.68 (2H, m, H-6'), $4.44(1 \mathrm{H}, \mathrm{t}, J .5 .6 \mathrm{~Hz},-\mathrm{OH}), 4.93(1 \mathrm{H}, \mathrm{d}, J .4 .5 \mathrm{~Hz},-\mathrm{OH}), 5.00(1 \mathrm{H}, \mathrm{d}, J .4 .4 \mathrm{~Hz},-\mathrm{OH}), 5.11(1 \mathrm{H}$, d, J.5.6 Hz, - OH), $5.87\left(2 \mathrm{H}, \mathrm{s},-\mathrm{NH}_{2}\right), 6.67(2 \mathrm{H}, \mathrm{m}, \mathrm{Ar}-\mathrm{H}), 6.72(2 \mathrm{H}, \mathrm{m}, \mathrm{H}-3, \mathrm{Ar}-\mathrm{H}), 7.53(2 \mathrm{H}$, $\mathrm{m}, \mathrm{H}-4, \mathrm{Ar}-\mathrm{H}) .{ }^{13} \mathrm{C}$ NMR $\left(50 \mathrm{MHz}, \mathrm{DMSO}-d_{6}+\mathrm{CDCl}_{3}\right): \delta_{\mathrm{C}} 39.9(\mathrm{C}-1), 61.6\left(\mathrm{C}-6^{\prime}\right), 70.7\left(\mathrm{C}-2^{\prime}\right)$, 
74.0 (C-3'), $76.4\left(\mathrm{C}-4^{\prime}\right), 78.6\left(\mathrm{C}-1 '^{\prime}\right), 81.0\left(\mathrm{C}^{\prime} 5^{\prime}\right), 113.2$ (Ar-C), 114.1 (Ar-C), 122.0 (Ar-C), 125.3 (C-3), 128.5 (Ar-C), 130.8 (Ar-C), 143.8 (C-4), 152.0 (Ar-C), 197.8 (C=O); HRMS: Calcd. Accurate mass for $\left(\mathrm{C}_{16} \mathrm{H}_{21} \mathrm{NNaO}_{6}\right): 346.1267$. Found $346.1249[\mathrm{M}+\mathrm{Na}]^{+}$.

2-[(1'-Deoxy-2',3', $\mathbf{4}^{\prime}$-tri- $\boldsymbol{O}$-acetyl- $\beta$-D-xylopyranos-1'-yl)methyl]quinoline $\quad(\mathbf{7 a}) . \quad$ It $\quad$ was obtained by the reaction of 2-nitrophenyl 2-oxobut-3-enyl xylopyranoside 3a (1.0 g, $2.22 \mathrm{mmol}$ ) with $\mathrm{SnCl}_{2} .2 \mathrm{H}_{2} \mathrm{O}(5.02 \mathrm{~g}, 22.27 \mathrm{mmol})$ as a yellow solid, yield $63 \%, 0.56 \mathrm{~g}, \mathrm{mp} \mathrm{143-144}{ }^{\circ} \mathrm{C} ; R_{\mathrm{f}}$ 0.5 (5:5, Hexane-EtOAc); $[\alpha]_{\mathrm{D}}{ }^{25}-34\left(\mathrm{c} 0.1, \mathrm{CHCl}_{3}\right)$; IR $\left(v_{\max }, \mathrm{cm}^{-1}\right): 3440,1640,1220,769 .{ }^{1} \mathrm{H}$ NMR $\left(300 \mathrm{MHz}, \mathrm{CDCl}_{3}+\mathrm{CCl}_{4}\right): \delta_{\mathrm{H}} 2.01\left(9 \mathrm{H}, \mathrm{m}, 3 \times-\mathrm{COCH}_{3}\right), 3.13(1 \mathrm{H}, \mathrm{m}, \mathrm{H}-1 \mathrm{~b}), 3.25(1 \mathrm{H}, \mathrm{m}$, H-1a), 3.85 (1H, m, H-1'), 4.08 (2H, m, H-5'), 4.90 (1H, m, H-2'), 4.97 (2H, t, J.6.2 Hz, H-3', H4'), $7.33(1 \mathrm{H}, \mathrm{d}, J .8 .3 \mathrm{~Hz}, \mathrm{Ar}-\mathrm{H}), 7.52(1 \mathrm{H}, \mathrm{t}, J .7 .5 \mathrm{~Hz}, \mathrm{Ar}-\mathrm{H}), 7.71(1 \mathrm{H}, \mathrm{t}, J .6 .9 \mathrm{~Hz}, \mathrm{Ar}-\mathrm{H}), 7.79$ $(1 \mathrm{H}, \mathrm{d}, J .8 .1 \mathrm{~Hz}, \mathrm{Ar}-\mathrm{H}), 8.08(2 \mathrm{H}, \mathrm{m}, \mathrm{Ar}-\mathrm{H}) .{ }^{13} \mathrm{C} \mathrm{NMR}\left(75 \mathrm{MHz}, \mathrm{CDCl}_{3}+\mathrm{CCl}_{4}\right): \delta_{\mathrm{C}} 20.9(3 \times-$ $\left.\mathrm{COCH}_{3}\right), 41.3\left(-\mathrm{CH}_{2}{ }^{-}\right), 66.9\left(\mathrm{C}^{-} 5^{\prime}\right), 69.8\left(\mathrm{C}-4^{\prime}\right), 72.2\left(\mathrm{C}-2^{\prime}\right), 74.9\left(\mathrm{C}-3^{\prime}\right), 76.5\left(\mathrm{C}-1^{\prime}\right), 122.6(\mathrm{Ar}-$ C), 126.0 (Ar-C), 126.9 (Ar-C),127.4 (Ar-C), 128.8 (Ar-C), 129.4 (Ar-C), 137.0 (Ar-C), 147.7 (Ar-C), $158.2\left(\right.$ Ar-C), 171.1, 170.2, $169.7\left(3 \times-\mathrm{COCH}_{3}\right)$; HRMS: Calcd. Accurate mass for $\left(\mathrm{C}_{21} \mathrm{H}_{26} \mathrm{~N}_{2} \mathrm{O}_{7}\right): 418.1740$. Found $418.1511\left[\mathrm{M}+\mathrm{NH}_{3}\right]^{+}$.

(E)-4-(3-Aminophenyl)-1-[1'-deoxy-2',3',4'-tri- $O$-acetyl- $\beta$-D-xylopyranos-1'-yl]but-3-en-2-

one (7b). It was obtained by the reaction of 3-nitrophenyl 2-oxobut-3-enyl xylopyranoside $\mathbf{3 b}$ $(1.0 \mathrm{~g}, 2.22 \mathrm{mmol})$ with $\mathrm{SnCl}_{2} .2 \mathrm{H}_{2} \mathrm{O}(5.02 \mathrm{~g}, 22.27 \mathrm{mmol})$ as a yellow solid, yield $67 \%, 0.6 \mathrm{~g}$, mp 93-94 ${ }^{\circ} \mathrm{C} ; R_{\mathrm{f}} 0.4\left(6: 4\right.$, Hexane-EtOAc); $[\alpha]_{\mathrm{D}}{ }^{25}-62\left(0.1, \mathrm{CHCl}_{3}\right) ; \mathrm{IR}\left(v_{\max }, \mathrm{cm}^{-1}\right): 3429(\mathrm{~N}-\mathrm{H})$, $1746(\mathrm{C}=\mathrm{O}), 1626(\mathrm{C}=\mathrm{C}), 1226,768 .{ }^{1} \mathrm{H} \mathrm{NMR}\left(300 \mathrm{MHz}, \mathrm{CDCl}_{3}+\mathrm{CCl}_{4}\right): \delta_{\mathrm{H}} 2.01(9 \mathrm{H}, \mathrm{s}, 3 \times-$ $\left.\mathrm{OCOCH}_{3}\right), 2.63\left(1 \mathrm{H}, \mathrm{dd}, J_{1} .2 .9 \mathrm{~Hz}, J_{2 .} 15.9 \mathrm{~Hz}, \mathrm{H}-1 \mathrm{~b}\right), 2.97\left(1 \mathrm{H}, \mathrm{dd}, J_{1} .8 .4 \mathrm{~Hz}, J_{2} .16 .0 \mathrm{~Hz}, \mathrm{H}-1 \mathrm{a}\right)$, 3.30 (1H, m, H-1'), 3.56-3.53 (2H, m, -NH $), 4.05$ (2H, m, H-5'), 4.98 (2H, m, H-2', H-4'), 5.21 $(1 \mathrm{H}, \mathrm{t}$, J.9.3 Hz, H-3'), 6.61 (1H, s, Ar-H), 6.69 (1H, d, J.7.9 Hz, Ar-H), 6.91-6.80 (2H, m, H-3, Ar-H), $7.16(1 \mathrm{H}, \mathrm{t}, J .7 .7 \mathrm{~Hz}, \mathrm{Ar}-\mathrm{H}), 7.45(1 \mathrm{H}, \mathrm{d}, J .16 .1 \mathrm{~Hz}, \mathrm{H}-4) .{ }^{13} \mathrm{C} \mathrm{NMR}(50 \mathrm{MHz}$, $\left.\mathrm{CDCl}_{3}+\mathrm{CCl}_{4}\right): \delta_{\mathrm{C}} 20.6\left(3 \times-\mathrm{OCOCH}_{3}\right), 42.4(\mathrm{C}-1), 66.6\left(\mathrm{C}-5^{\prime}\right), 69.2\left(\mathrm{C}-4^{\prime}\right), 71.9\left(\mathrm{C}-2^{\prime}\right), 73.7(\mathrm{C}-$ 3'), 74.7 (C-1'), 114.1 (Ar-C), 117.4 (Ar-C), 118.9 (Ar-C), 128.0 (C-3), 129.7 (Ar-C), 135.1 (ArC), 144.0 (C-4), 146.9 (Ar-C), 169.5, 169.7, 169.9 (3 × - $\left.\mathrm{OCOCH}_{3}\right), 196.1$ (C=O); HRMS: Calcd. Accurate mass for $\left(\mathrm{C}_{21} \mathrm{H}_{25} \mathrm{NNaO}_{8}\right): 442.1478$. Found $442.1458[\mathrm{M}+\mathrm{Na}]^{+}$.

(E)-4-(4-Aminophenyl)-1-[1'-deoxy-2',3',4'-tri- $\boldsymbol{O}$-acetyl- $\beta$-D-xylopyranos-1'-yl]but-3-en-2-

one (7c). It was obtained by the reaction of 4-nitrophenyl 2-oxobut-3-enyl xylopyranoside 3c $(1.0 \mathrm{~g}, 2.22 \mathrm{mmol})$ with $\mathrm{SnCl}_{2} .2 \mathrm{H}_{2} \mathrm{O}(5.02 \mathrm{~g}, 22.27 \mathrm{mmol})$ as a yellow solid, yield 69\%, $0.64 \mathrm{~g}$, mp 167-169 ${ }^{\circ} \mathrm{C} ; R_{\mathrm{f}} 0.4\left(6: 4\right.$, Hexane-EtOAc); $[\alpha]_{\mathrm{D}}{ }^{25}-78\left(\mathrm{c}, 0.1, \mathrm{CHCl}_{3}\right) ; \mathrm{IR}\left(v_{\max }, \mathrm{cm}^{-1}\right): 3354(\mathrm{~N}-$ $\mathrm{H}), 1739(\mathrm{C}=\mathrm{O}), 1635(\mathrm{C}=\mathrm{C}), 1225,772 .{ }^{1} \mathrm{H} \mathrm{NMR}\left(300 \mathrm{MHz}, \mathrm{CDCl}_{3}+\mathrm{CCl}_{4}\right): \delta_{\mathrm{H}} 2.04(9 \mathrm{H}, \mathrm{m}, 3$ $\left.\times-\mathrm{OCOCH}_{3}\right), 2.62(1 \mathrm{H}, \mathrm{d}, J .15 .2 \mathrm{~Hz}, \mathrm{H}-1 \mathrm{~b}), 2.96\left(1 \mathrm{H}, \mathrm{dd}, J_{1} .7 .2 \mathrm{~Hz}, J_{2} .14 .3 \mathrm{~Hz}, \mathrm{H}-1 \mathrm{a}\right), 3.37$ (1H, m, H-1'), 4.14 (2H, m, H-5'), 4.99 (2H, m, H-2', H-4'), 5.23 (1H, t, J.9.4 Hz, H-3'), 6.58 $(1 \mathrm{H}, \mathrm{d}, J .15 .9 \mathrm{~Hz}, \mathrm{H}-3), 6.66(2 \mathrm{H}, \mathrm{m}, \mathrm{Ar}-\mathrm{H}), 7.39(2 \mathrm{H}, \mathrm{m}, J .7 .7 \mathrm{~Hz}, \mathrm{Ar}-\mathrm{H}), 7.50(1 \mathrm{H}, \mathrm{d}, J .16 .2$ $\mathrm{Hz}, \mathrm{H}-4) .{ }^{13} \mathrm{C} \mathrm{NMR}\left(50 \mathrm{MHz}, \mathrm{CDCl}_{3}+\mathrm{CCl}_{4}\right): \delta_{\mathrm{C}} 20.6\left(3 \times-\mathrm{OCOCH}_{3}\right), 42.4(\mathrm{C}-1), 66.7\left(\mathrm{C}-5^{\prime}\right)$, $69.3\left(\mathrm{C}-2^{\prime}\right), 72.0\left(\mathrm{C}^{\prime} 4^{\prime}\right), 73.8\left(\mathrm{C}-3^{\prime}\right), 75.0\left(\mathrm{C}-1^{\prime}\right), 114.7$ (Ar-C), 122.3 (Ar-C), 124.4 (C-3), 130.4 (Ar-C), 144.1 (C-4), 149.1 (Ar-C), 169.5, 169.7, 169.9 (3 $\left.\times-\mathrm{OCOCH}_{3}\right), 195.8(\mathrm{C}=\mathrm{O})$; HRMS: Calcd. Accurate mass for $\left(\mathrm{C}_{21} \mathrm{H}_{25} \mathrm{NNaO}_{8}\right): 442.1478$. Found $442.1461[\mathrm{M}+\mathrm{Na}]^{+}$. 
2-[(1'-Deoxy-2', 3', $\mathbf{4}^{\prime}, \mathbf{6}^{\prime}$-tetra- $\boldsymbol{O}$-acetyl- $\boldsymbol{\beta}$-D-glucopyranos-1'-yl)methyl]quinoline (8a). It was obtained by the reaction of 2-nitrophenyl 2-oxobut-3-enyl glucopyranoside 4a (1.0 g, 1.91 mmol) with $\mathrm{SnCl}_{2} .2 \mathrm{H}_{2} \mathrm{O}(4.33 \mathrm{~g}, 19.19 \mathrm{mmol})$ as a light yellow solid, yield $62 \%, 0.56 \mathrm{~g}$, mp $117-119{ }^{\circ} \mathrm{C} ; R_{\mathrm{f}} 0.5$ (5:5, Hexane-EtOAc); $[\alpha]_{\mathrm{D}}{ }^{25}-5\left(\mathrm{c} 0.1, \mathrm{CHCl}_{3}\right)$; IR $\left(v_{\max }, \mathrm{cm}^{-1}\right): 3318,1752$, 1235, 769. ${ }^{1} \mathrm{H} \mathrm{NMR}\left(300 \mathrm{MHz}, \mathrm{CDCl}_{3}+\mathrm{CCl}_{4}\right): \delta_{\mathrm{H}} 2.02-1.93\left(12 \mathrm{H}, \mathrm{m}, 4 \times-\mathrm{OCOCH}_{3}\right), 3.18(2 \mathrm{H}$, m, H-1), 3.60 (1H, m, H-1'), 3.99 (1H, m, H-6'b), 4.19 (1H, m, H-6'a), 4.23 (1H, m, H-5'), 5.09 (2H, m, H-2', H-4'), 5.24 (1H, t, J.9.0 Hz, H-3'), 7.35 (1H, d, J.8.31 Hz, Ar-H), 7.53 (1H, t, J.7.0 $\mathrm{Hz}, \mathrm{Ar}-\mathrm{H}), 7.72\left(1 \mathrm{H}, \mathrm{t}\right.$, J.7.0 Hz, Ar-H), $7.79\left(1 \mathrm{H}, \mathrm{d}\right.$, J.7.7 Hz, Ar-H), $8.07(2 \mathrm{H}, \mathrm{m}, \mathrm{Ar}-\mathrm{H}) .{ }^{13} \mathrm{C}$ NMR $\left(50 \mathrm{MHz}, \mathrm{CDCl}_{3}+\mathrm{CCl}_{4}\right): \delta_{\mathrm{C}} 20.5\left(4 \times-\mathrm{OCOCH}_{3}\right), 41.1(\mathrm{C}-1), 62.0\left(\mathrm{C}-6^{\prime}\right), 68.7\left(\mathrm{C}-4^{\prime}\right)$, 72.1 (C-3'), 74.3 (C-2'), 75.7 (C-1'), 76.3 (C-5'), 122.8 (Ar-C), 126.0 (Ar-C), 126.9 (Ar-C), 127.4 (Ar-C), 128.9 (Ar-C), 129.4 (Ar-C), 135.8 (Ar-C), 147.8 (Ar-C), 157.8 (Ar-C), 173.8, 170.1, 169.5, $169.2\left(4 \times-\mathrm{OCOCH}_{3}\right)$; HRMS: Calcd. Accurate mass for $\left(\mathrm{C}_{24} \mathrm{H}_{28} \mathrm{NO}_{9}\right)$ : 474.1764. Found $474.1762[\mathrm{M}+\mathrm{H}]^{+}$.

(E)-4-(3-Aminophenyl)-1-[1'-deoxy-2',3',4',6'-tetra- $O$-acetyl- $\beta$-D-glucopyranos-1'-yl]-but-3en-2-one $(\mathbf{8 b})$. It was obtained by the reaction of 3-nitrophenyl 2-oxobut-3-enyl glucopyranoside $4 \mathbf{b}(1.0 \mathrm{~g}, 1.91 \mathrm{mmol})$ with $\mathrm{SnCl}_{2} .2 \mathrm{H}_{2} \mathrm{O}(4.33 \mathrm{~g}, 19.19 \mathrm{mmol})$ as a light yellow solid, yield $68 \%$, $0.64 \mathrm{~g}, \mathrm{mp} 107-109{ }^{\circ} \mathrm{C} ; R_{\mathrm{f}} 0.4\left(5: 5\right.$, Hexane-EtOAc); $[\alpha]_{\mathrm{D}}{ }^{25}-24\left(\mathrm{c} 0.1, \mathrm{CHCl}_{3}\right) ; \mathrm{IR}\left(v_{\max }, \mathrm{cm}^{-1}\right)$ : $3378(\mathrm{~N}-\mathrm{H}), 1748(\mathrm{C}=\mathrm{O}), 1655(\mathrm{C}=\mathrm{C}), 1221,771 .{ }^{1} \mathrm{H} \mathrm{NMR}\left(300 \mathrm{MHz}, \mathrm{CDCl}_{3}+\mathrm{CCl}_{4}\right): \delta_{\mathrm{H}} 2.02$ $\left(12 \mathrm{H}, \mathrm{m}, 4 \times-\mathrm{OCOCH}_{3}\right), 2.68\left(1 \mathrm{H}, \mathrm{dd}, J_{1} .2 .0 \mathrm{~Hz}, J_{2} .16 .1 \mathrm{~Hz}, \mathrm{H}-1 \mathrm{~b}\right), 3.02\left(1 \mathrm{H}, \mathrm{dd}, J_{1} .8 .3 \mathrm{~Hz}\right.$, $\left.J_{2} .16 .1 \mathrm{~Hz}, \mathrm{H}-1 \mathrm{a}\right), 3.72-3.67$ (3H, m, -NH $\left.2, \mathrm{H}^{-1}\right)$, 4.13-3.99 (2H, m, H-6'), 4.29-4.23 (1H, m, H5'), 4.99 (1H, t, J.9.6 Hz, H-4'), 5.08 (1H, t, J.9.7 Hz, H-2'), 5.23 (1H, t, J.9.2 Hz, H-3'), 6.68 $(1 \mathrm{H}, \mathrm{d}, J .16 .1 \mathrm{~Hz}, \mathrm{H}-3), 6.71(1 \mathrm{H}, \mathrm{m}, \mathrm{Ar}-\mathrm{H}), 6.82(1 \mathrm{H}, \mathrm{s}, \mathrm{Ar}-\mathrm{H}), 6.93(1 \mathrm{H}, \mathrm{d}, J .7 .4 \mathrm{~Hz}, \mathrm{Ar}-\mathrm{H})$, $7.18(1 \mathrm{H}, \mathrm{t}, J .7 .7 \mathrm{~Hz}, \mathrm{Ar}-\mathrm{H}), 7.46(1 \mathrm{H}, \mathrm{d}, J .16 .1 \mathrm{~Hz}, \mathrm{H}-4) .{ }^{13} \mathrm{C} \mathrm{NMR}\left(50 \mathrm{MHz}, \mathrm{CDCl}_{3}+\mathrm{CCl}_{4}\right): \delta_{\mathrm{C}}$ 20.6-20.5 (4 ×- $\left.\mathrm{OCOCH}_{3}\right), 42.6(\mathrm{C}-1), 61.9\left(\mathrm{C}-6^{\prime}\right), 68.4\left(\mathrm{C}-2^{\prime}\right), 71.7\left(\mathrm{C}-4^{\prime}\right), 74.2\left(\mathrm{C}-3^{\prime}\right), 75.7(\mathrm{C}-$ $1^{\prime}$ ), 76.3 (C-5'), 114.0 (Ar-C), 117.4 (Ar-C), 118.9 (Ar-C), 126.0 (C-3), 129.8 (Ar-C), 135.2 (ArC), 144.0 (C-4), 146.9 (Ar-C), 169.1, 169.5, 169.8, 170.2 (4 × - $\left.\mathrm{OCOCH}_{3}\right), 195.7$ (C=O); HRMS: Calcd. Accurate mass for $\left(\mathrm{C}_{24} \mathrm{H}_{29} \mathrm{NNaO}_{10}\right): 514.1689$. Found 514.1662 [M+Na] ${ }^{+}$.

(E)-4-(4-Aminophenyl)-1-[1'-deoxy-2',3',4',6'-tetra- $O$-acetyl- $\beta$-D-glucopyranos-1'-yl]-but-3en-2-one (8c). It was obtained by the reaction of 4-nitrophenyl 2-oxobut-3-enyl glucopyranoside $4 c(1.0 \mathrm{~g}, 1.91 \mathrm{mmol})$ with $\mathrm{SnCl}_{2} .2 \mathrm{H}_{2} \mathrm{O}(4.33 \mathrm{~g}, 19.19 \mathrm{mmol})$ as a yellow solid, yield $69 \%, 0.65$ $\mathrm{g}, \mathrm{mp} 128-130{ }^{\circ} \mathrm{C} ; R_{\mathrm{f}} 0.4\left(5: 5\right.$, Hexane-EtOAc); $[\alpha]_{\mathrm{D}}{ }^{25}-8\left(\mathrm{c} 0.1, \mathrm{CHCl}_{3}\right) ; \mathrm{IR}\left(v_{\max }, \mathrm{cm}^{-1}\right): 3372$ $(\mathrm{N}-\mathrm{H}), 1748(\mathrm{C}=\mathrm{O}), 1588,1230,759 .{ }^{1} \mathrm{H} \mathrm{NMR}\left(300 \mathrm{MHz}, \mathrm{CDCl}_{3}+\mathrm{CCl}_{4}\right): \delta_{\mathrm{H}} 2.02(12 \mathrm{H}, \mathrm{m}, 4 \times-$ $\left.\mathrm{OCOCH}_{3}\right), 2.66\left(1 \mathrm{H}, \mathrm{dd}, J_{1} .3 .2 \mathrm{~Hz}, J_{2} .16 .1 \mathrm{~Hz}, \mathrm{H}-1 \mathrm{~b}\right), 3.00\left(1 \mathrm{H}, \mathrm{dd}, J_{1} .8 .3 \mathrm{~Hz}, J_{2} .16 .1 \mathrm{~Hz}, \mathrm{H}-\right.$ 1a), 3.73 (2H, m, -NH $)$, 4.11-3.98 (3H, m, H-6', H-1'), 4.29 (1H, m, H-5'), 5.00 (1H, t, J.9.7 Hz, H-4'), 5.09 (1H, t, J.9.7 Hz, H-2'), 5.23 (1H, t, J.9.3 Hz, H-3'), 6.58 (1H, d, J.7.6 Hz, H-3), 6.65 $\left(2 \mathrm{H}, \mathrm{d}\right.$, J.7.5 Hz, Ar-H), $7.38(2 \mathrm{H}, \mathrm{d}, J .7 .7 \mathrm{~Hz}, \mathrm{Ar}-\mathrm{H}), 7.49(1 \mathrm{H}, \mathrm{d}, J .16 .1 \mathrm{~Hz}, \mathrm{H}-4) .{ }^{13} \mathrm{C}$ NMR $(75$ $\left.\mathrm{MHz}, \mathrm{CDCl}_{3}+\mathrm{CCl}_{4}\right): \delta_{\mathrm{C}} 20.5\left(4 \times-\mathrm{OCOCH}_{3}\right), 42.4(\mathrm{C}-1), 61.9\left(\mathrm{C}-6^{\prime}\right), 68.5\left(\mathrm{C}-2^{\prime}\right), 71.8\left(\mathrm{C}-4^{\prime}\right)$, 74.2 (C-3'), 74.3 (C-1'), 75.7 (C-5'), 114.7 (Ar-C), 122.2 (C-3), 124.4 (Ar-C), 130.4 (C-4), 144.1 (Ar-C), 169.2, 169.6, 169.8, $170.2\left(4 \times-\mathrm{OCOCH}_{3}\right), 195.4(\mathrm{C}=\mathrm{O})$; HRMS: Calcd. Accurate mass for $\left(\mathrm{C}_{24} \mathrm{H}_{29} \mathrm{NNaO}_{10}\right): 514.1689$. Found 514.1677 [M+Na] ${ }^{+}$. 
(E)-4-(3-Nitrophenyl)-1-[1'-deoxy-2',3',4'-tri- $O$-acetyl-6' $-O$-(p-toluenesulfonyl)- $\beta$-D-glucopyranos-1'-yl]but-3-en-2-one (9). To a stirring solution of 3-nitrophenyl-2-oxobut-3-enyl glucopyranoside (2a) $(1.00 \mathrm{~g}, 2.83 \mathrm{mmol})$ in pyridine, $\mathrm{Et}_{3} \mathrm{~N}(0.078 \mathrm{~mL}, 0.056 \mathrm{mmol})$ was added and solution was cooled to $0{ }^{\circ} \mathrm{C}$. Tosyl chloride $(\mathrm{TsCl})(0.64 \mathrm{~g}, 3.39 \mathrm{mmol})$ was gradually added to the stirring solution. After addition of $p$-toluenesulfonyl chloride the reaction mixture and stirring was continued at the same temperature till the starting sugar is consumed totally (TLC). After completion of the reaction, acetic anhydride $\left(\mathrm{Ac}_{2} \mathrm{O}\right)(0.43 \mathrm{~mL}, 3.11 \mathrm{mmol})$ was added (dropwise) to the stirring reaction mixture at $0{ }^{\circ} \mathrm{C}$ followed by stiriing at room temperature till the reaction was completed (TLC). The reaction mixture was partitioned between ethylacetate and water and organic layer was washed with water and dried $\left(\mathrm{Na}_{2} \mathrm{SO}_{4}\right)$ and the solvent was evaporated under reduced pressure to give a crude mass, which was purified by column chromatography $\left(\mathrm{SiO}_{2} 60-120\right)$ using hexan:ethylacetate (3:1) as eluent to give the compound 9 as white solid, Yield $78 \%, 1.40 \mathrm{~g}, \mathrm{mp} 123-125^{\circ} \mathrm{C} ; R_{\mathrm{f}} 0.5\left(5: 5\right.$, Hexane-EtOAc); $[\alpha]_{\mathrm{D}}{ }^{25}-13.4(\mathrm{c}$ $\left.0.1, \mathrm{CHCl}_{3}\right)$; IR $\left(v_{\max }, \mathrm{cm}^{-1}\right): 2943,1753(\mathrm{C}=\mathrm{O}), 1604(\mathrm{C}=\mathrm{C}), 1521,1361(\mathrm{~N}-\mathrm{O}), 1241,827 .{ }^{1} \mathrm{H}$ NMR $\left(300 \mathrm{MHz}, \mathrm{CDCl}_{3}\right): \delta_{\mathrm{H}} 2.01-1.96\left(9 \mathrm{H}, \mathrm{m}, 3 \times-\mathrm{COCH}_{3}\right), 2.42\left(3 \mathrm{H}, \mathrm{s}, \mathrm{CH}_{3}\right), 2.70(1 \mathrm{H}, \mathrm{dd}$, $\left.J_{1} .16 .2 \mathrm{~Hz}, J_{2} .2 .94 \mathrm{~Hz}\right), 3.01\left(1 \mathrm{H}, \mathrm{dd}, J_{1} .16 .3 \mathrm{~Hz}, J_{2} .8 .3 \mathrm{~Hz}\right), 3.74-3.70(1 \mathrm{H}, \mathrm{m}), 4.11-3.99(3 \mathrm{H}$, m), $4.95\left(1 \mathrm{H}, \mathrm{t}, J .9 .9 \mathrm{~Hz}, \mathrm{H}-2^{\prime}\right), 5.02\left(1 \mathrm{H}, \mathrm{t}, J .9 .7 \mathrm{~Hz}, \mathrm{H}-4^{\prime}\right), 5.22\left(1 \mathrm{H}, \mathrm{t}, J .9 .3 \mathrm{~Hz}, \mathrm{H}-3^{\prime}\right), 6.88$ $(1 \mathrm{H}, \mathrm{d}, J .16 .1 \mathrm{~Hz}, \mathrm{H}-3), 7.32$ (2H, d, J.8.0 Hz, Ar-H), 7.64-7.55 (3H, m, 2 x Ar-H, H-4), 7.73 $(2 \mathrm{H}, \mathrm{d}, J .8 .1 \mathrm{~Hz}, \mathrm{Ar}-\mathrm{H}), 7.90(1 \mathrm{H}, \mathrm{d}, J .7 .5 \mathrm{~Hz}, \mathrm{Ar}-\mathrm{H}), 8.27(1 \mathrm{H}, \mathrm{d}, J .8 .0 \mathrm{~Hz}, \mathrm{Ar}-\mathrm{H}), 8.41(1 \mathrm{H}, \mathrm{s}$, $\mathrm{Ar}-\mathrm{H}) .{ }^{13} \mathrm{C} \mathrm{NMR}\left(75 \mathrm{MHz}, \mathrm{CDCl}_{3}\right): \delta_{\mathrm{C}} 20.6,20.6,20.5\left(3 \times-\mathrm{OCOCH}_{3}\right), 21.6\left(\mathrm{CH}_{3}\right), 42.9(\mathrm{C}-1)$, 67.3 (C-6'), 68.4 (C-2'), 71.3 (C-4'), 73.8 (C-3'), 74.0 (C-1'), 75.1 (C-5'), 122.7 (Ar-C), 124.9 (Ar-C), 128.0 (Ar-C), 128.4 (Ar-C), 129.8 (Ar-C), 130.1 (Ar-C), 132.4 (Ar-C), 134.0 (C-3), 136.0 (Ar-C), 140.6 (Ar-C), 145.0 (C-4), 148.7 (Ar-C), 169.3, 169.8, $170.2\left(3 \times-\mathrm{COCH}_{3}\right), 195.5$ $(\mathrm{C}=\mathrm{O})$; HRMS: Calcd. Accurate mass for $\left(\mathrm{C}_{29} \mathrm{H}_{31} \mathrm{NNaO}_{13} \mathrm{~S}\right)$ : 656.1414. Found 656.1412 $[\mathrm{M}+\mathrm{Na}]^{+}$.

(E)-4-(3-Nitrophenyl)-1-[6' -azido-1', 6' -dideoxy-2',3',4'-tri- $O$-acetyl- $\beta$-D-glucopyranos-1'-

yl]but-3-en-2-one (10). To a stirring solution of $(E)$-4-(3-nitrophenyl)-1-[1'-deoxy-2',3',4'-tri- $O$ acetyl-6'-O-(4-methyl-benzenesulfonyl)- $\beta$-D-glucopyranos-1'-yl]but-3-en-2-one (9) (0.8 g, 1.26 mmol $)$ in DMF $(15 \mathrm{ml})$ was added $\mathrm{NaN}_{3}(0.09 \mathrm{~g}, 1.38 \mathrm{mmol})$ and the reaction mixture was stirred at $80{ }^{\circ} \mathrm{C}$ until completion (TLC) of reaction. The reaction mixture was partitioned between ethylacetate and water and organic layer was separated and dried (anhyd $\mathrm{Na}_{2} \mathrm{SO}_{4}$ ) and evaporated under reduced pressure to give a crude mass. The latter was purified by column chromatography $\left(\mathrm{SiO}_{2} 60-120\right)$ using hexane : ethyl acetate (5:1) as eluent to give the compound 10 as colourless solid, Yield 53\%, $0.34 \mathrm{~g}, \mathrm{mp} 141-143{ }^{\circ} \mathrm{C} ; R_{\mathrm{f}} 0.5$ (6.5:4.5, Hexane-EtOAc); $[\alpha]_{\mathrm{D}}^{25}-7.6\left(\mathrm{c} 0.1, \mathrm{CHCl}_{3}\right) ; \mathrm{IR}\left(v_{\max }, \mathrm{cm}^{-1}\right): 2939,2100,1743(\mathrm{C}=\mathrm{O}), 1614(\mathrm{C}=\mathrm{C}), 1548,1376$ $(\mathrm{N}-\mathrm{O}), 1228,769 .{ }^{1} \mathrm{H}$ NMR $\left(300 \mathrm{MHz}, \mathrm{CDCl}_{3}\right): \delta_{\mathrm{H}} 2.03-1.97\left(9 \mathrm{H}, \mathrm{m}, 3 \times-\mathrm{COCH}_{3}\right), 2.72(1 \mathrm{H}$, dd, $\left.J_{1} .16 .2 \mathrm{~Hz}, J_{2} .3 .0 \mathrm{~Hz}\right), 3.02\left(1 \mathrm{H}, \mathrm{dd}, J_{1} .16 .2 \mathrm{~Hz}, J_{2} .8 .4 \mathrm{~Hz}\right), 3.27-3.20(2 \mathrm{H}, \mathrm{m}), 3.76-3.72$ (1H, m), 4.13-4.09 (1H, m), 5.04-4.90 (2H, m, H-4', H-2'), 5.23 (1H, t, J.9.2 Hz, H-3'), 6.89 (1H, d, J.15.8 Hz, H-3), 7.66-7.57 (3H, m, 2 x Ar-H, H-4), 7.91 (1H, d, J.7.5 Hz, Ar-H), 8.28 (1H, d, J.8.1 Hz, Ar-H), $8.42(1 \mathrm{H}, \mathrm{s}, \mathrm{Ar}-\mathrm{H}) .{ }^{13} \mathrm{C} \mathrm{NMR}\left(75 \mathrm{MHz}, \mathrm{CDCl}_{3}\right): \delta_{\mathrm{C}} 20.7,20.6,20.5(3 \times-$ 
$\left.\mathrm{OCOCH}_{3}\right), 42.9(\mathrm{C}-1), 50.9\left(\mathrm{C}-6^{\prime}\right), 69.4\left(\mathrm{C}-2^{\prime}\right), 71.5\left(\mathrm{C}-4^{\prime}\right), 73.9\left(\mathrm{C}-3^{\prime}\right), 74.0\left(\mathrm{C}-1^{\prime}\right), 75.2\left(\mathrm{C}-5^{\prime}\right)$, 122.9 (Ar-C), 128.5 (Ar-C), 132.5 (Ar-C), 134.0 (C-3), 136.0 (Ar-C), 140.6 (C-4), 148.7 (Ar-C), 169.5, 169.9, $170.2\left(3 \times-\mathrm{COCH}_{3}\right), 195.6(\mathrm{C}=\mathrm{O})$; HRMS: Calcd. Accurate mass for $\left(\mathrm{C}_{22} \mathrm{H}_{24} \mathrm{~N}_{4} \mathrm{NaO}_{10}\right): 527.1390$. Found 527.1384 [M+Na] ${ }^{+}$.

(E)-4-(3-Aminophenyl)-1-[1'-deoxy-2',3',4'-tri- $O$-acetyl-6' $O$ - $(\boldsymbol{p}$-toluenesulfonyl)- $\beta$-D-glucopyranos-1'-yl]but-3-en-2-one (11). It was obtained by the reaction of (E)-4-(3-nitrophenyl)-1[1'-deoxy-2',3',4'-tri- $O$-acetyl-6'-O-(4-methyl benzenesulphonyl)- $\beta$-D-glucopyranos-1'-yl]but-3en-2-one (9) $(0.5 \mathrm{~g}, 0.79 \mathrm{mmol})$ with $\mathrm{SnCl}_{2} .2 \mathrm{H}_{2} \mathrm{O}(1.78 \mathrm{~g}, 7.90 \mathrm{mmol})$ as described for compound $8 \mathrm{~b}$ to give compound 11 as a light yellow solid, yield $63 \%, 0.3 \mathrm{~g}, \mathrm{mp} 134-135{ }^{\circ} \mathrm{C} ; R_{\mathrm{f}}$ 0.5 (5:5, Hexane-EtOAc); $[\alpha]_{\mathrm{D}}{ }^{25}-4.7\left(\mathrm{c} 0.1, \mathrm{CHCl}_{3}\right)$; IR $\left(v_{\max }, \mathrm{cm}^{-1}\right): 3421(\mathrm{~N}-\mathrm{H}), 1761(\mathrm{C}=\mathrm{O})$, $1610(\mathrm{C}=\mathrm{C}), 1241,767 .{ }^{1} \mathrm{H}$ NMR $\left(300 \mathrm{MHz}, \mathrm{CDCl}_{3}\right): \delta_{\mathrm{H}} 1.99-1.97\left(9 \mathrm{H}, \mathrm{m}, 3 \times-\mathrm{COCH}_{3}\right), 2.40$ $\left(3 \mathrm{H}, \mathrm{s}, \mathrm{CH}_{3}\right), 2.60\left(1 \mathrm{H}, \mathrm{dd}, J_{1} .16 .0 \mathrm{~Hz}, J_{2} .3 .0 \mathrm{~Hz}\right), 2.89\left(1 \mathrm{H}, \mathrm{dd}, J_{1} .16 .0 \mathrm{~Hz}, J_{2} .8 .10 \mathrm{~Hz}\right), 3.08$ $\left(2 \mathrm{H}, \mathrm{bs}, \mathrm{NH}_{2}\right), 3.71-3.67(1 \mathrm{H}, \mathrm{m}), 4.09-3.97(3 \mathrm{H}, \mathrm{m}), 4.90\left(1 \mathrm{H}, \mathrm{t}, J .9 .6 \mathrm{~Hz}, \mathrm{H}-2^{\prime}\right), 4.99(1 \mathrm{H}, \mathrm{t}$, J.9.8 Hz, H-4'), 5.16 (1H, t, J.9.2 Hz, H-3'), $6.67(1 \mathrm{H}, \mathrm{d}, J .16 .1 \mathrm{~Hz}, \mathrm{H}-3), 6.74$ (1H, d, J.7.7 Hz, Ar-H), 6.94-6.88 (2H, m, Ar-H), $7.19(1 \mathrm{H}, \mathrm{t}, J .7 .62 \mathrm{~Hz}, \mathrm{Ar}-\mathrm{H}), 7.27(2 \mathrm{H}, \mathrm{d}$, J.7.5 Hz, Ar-H), $7.45(1 \mathrm{H}, \mathrm{d}, J .16 .1 \mathrm{~Hz}, \mathrm{H}-4), 7.72(2 \mathrm{H}, \mathrm{d}, J .8 .1 \mathrm{~Hz}, \mathrm{Ar}-\mathrm{H}) .{ }^{13} \mathrm{C} \mathrm{NMR}\left(75 \mathrm{MHz}, \mathrm{CDCl}_{3}\right): \delta_{\mathrm{C}} 21.6$ $\left(\mathrm{CH}_{3}\right), 20.6,20.6,20.5\left(3 \times-\mathrm{OCOCH}_{3}\right), 42.2(\mathrm{C}-1), 67.2\left(\mathrm{C}-6^{\prime}\right), 68.4\left(\mathrm{C}-2^{\prime}\right), 71.3\left(\mathrm{C}-4^{\prime}\right), 73.8$ (C-3'), 74.0 (C-1'), 75.1 (C-5'), 113.8 (Ar-C), 114.5 (Ar-C), 116.5 (Ar-C), 126.3 (Ar-C), 128.0 (Ar-C), 129.8 (Ar-C), 130.1 (Ar-C), 132.5 (Ar-C), 135.0 (C-3), 136.0 (Ar-C), 140.6 (Ar-C), 144.9 (C-4), 148.6 (Ar-C), 169.2, 169.8, 170.2 (3 × - $\left.\mathrm{COCH}_{3}\right), 195.6(\mathrm{C}=\mathrm{O})$; ESIMS: m/z626 $[\mathrm{M}+\mathrm{Na}]^{+}$, molecular formula: $\mathrm{C}_{29} \mathrm{H}_{33} \mathrm{NO}_{11} \mathrm{~S}$.

(E)-4-(3-Aminophenyl)-1-[6'-azido-1',6'-dideoxy-2',3',4'-tri- $O$-acetyl- $\beta$-D-glucopyranos-1'yl]but-3-en-2-one (12). It was obtained by the reduction of $(E)-4-(3-n i t r o p h e n y l)-1-\left[6^{\prime}\right.$-azido1',6'-dideoxy-2',3',4'-tri- $O$-acetyl- $\beta$-D-glucopyranos-1'-yl]but-3-en-2-one 10 (0.3 g, $0.59 \mathrm{mmol})$ with $\mathrm{SnCl}_{2} .2 \mathrm{H}_{2} \mathrm{O}(1.34 \mathrm{~g}, 5.94 \mathrm{mmol})$ as above to give compound 12 a light yellow solid, yield $61 \%, 0.17 \mathrm{~g}, \mathrm{mp} 153-155{ }^{\circ} \mathrm{C} ; R_{\mathrm{f}} 0.5$ (4:6, Hexane-EtOAc); $[\alpha]_{\mathrm{D}}{ }^{25}-6.3\left(\mathrm{c} 0.1, \mathrm{CHCl}_{3}\right) ; \mathrm{IR}\left(v_{\max }\right.$, $\left.\mathrm{cm}^{-1}\right): 3376(\mathrm{~N}-\mathrm{H}), 2103,1752(\mathrm{C}=\mathrm{O}), 1620(\mathrm{C}=\mathrm{C}), 1232,688 .{ }^{1} \mathrm{H}$ NMR $\left(300 \mathrm{MHz}, \mathrm{CDCl}_{3}\right): \delta_{\mathrm{H}}$ 1.99-1.97 (9H, m, $\left.3 \times-\mathrm{COCH}_{3}\right), 2.61\left(1 \mathrm{H}, \mathrm{dd}, J_{1} .15 .9 \mathrm{~Hz}, J_{2} .3 .0 \mathrm{~Hz}\right), 2.89\left(1 \mathrm{H}, \mathrm{dd}, J_{1} .15 .9 \mathrm{~Hz}\right.$, $\left.J_{2} .8 .4 \mathrm{~Hz}\right), 3.25-3.14\left(4 \mathrm{H}, \mathrm{m}, 2 \times \mathrm{CH}, \mathrm{NH}_{2}\right), 3.71-3.67(1 \mathrm{H}, \mathrm{m}), 4.10-4.05(1 \mathrm{H}, \mathrm{m}), 4.90(1 \mathrm{H}, \mathrm{t}$, J.9.9 Hz, H-2'), 4.99 (1H, t, J.9.9 Hz, H-4'), 5.16 (1H, t, J.8.8 Hz, H-3'), 6.67 (1H, d, J.16.5 Hz, H-3), 6.73 (1H, d, J.7.1 Hz, Ar-H), 6.93-6.88 (2H, m, Ar-H),7.19 (1H, t, J.7.1 Hz, Ar-H), 7.45 $(1 \mathrm{H}, \mathrm{d}, \mathrm{J} .16 .5 \mathrm{~Hz}, \mathrm{H}-4) .{ }^{13} \mathrm{C} \mathrm{NMR}\left(75 \mathrm{MHz}, \mathrm{CDCl}_{3}\right): \delta_{\mathrm{C}} 20.5,20.4,20.4\left(3 \times-\mathrm{COCH}_{3}\right), 42.8(\mathrm{C}-$ 1), 50.7 (C-6'), $68.3\left(\mathrm{C}-2^{\prime}\right), 71.2\left(\mathrm{C}^{\prime} 4^{\prime}\right), 73.7$ (C-3'), 73.9 (C-1'), 75.0 (C-5'), 113.4 (Ar-C), 114.2 (Ar-C), 115.6 (Ar-C), 126.0 (Ar-C), 128.4 (Ar-C), 141.0 (Ar-C), 142.7 (C-4), 148.6 (Ar-C), 169.2, 169.7, $170.1\left(3 \times-\mathrm{COCH}_{3}\right), 195.4(\mathrm{C}=\mathrm{O})$; HRMS: Calcd. Accurate mass for $\left(\mathrm{C}_{22} \mathrm{H}_{28} \mathrm{~N}_{4} \mathrm{O}_{8}\right): 475.1829$. Found $475.1827[\mathrm{M}+\mathrm{H}]^{+}$.

(E)-1-[3-( $p$-Toluenesulfonamido)phenyl]-4-[1'-deoxy-2',3',4',6'-tetra- $O$-acetyl- $\beta$-D-glucopyranos-1'-yl]but-3-en-2-one (13). To a stirring solution of (E)-4-(3-aminophenyl)-1-[1'-deoxy$2^{\prime}, 3^{\prime}, 4^{\prime}, 6^{\prime}$-tetra- $O$-acetyl- $\beta$-D-glucopyranos-1'-yl]but-3-en-2-one $8 \mathrm{~b}(0.5 \mathrm{~g}, 1.01 \mathrm{mmol})$ in $\mathrm{CH}_{2} \mathrm{Cl}_{2}$ $(15.0 \mathrm{~mL})$ at $0{ }^{\circ} \mathrm{C}, \mathrm{Et}_{3} \mathrm{~N}(\mathrm{ml} 1.01 \mathrm{mmol})$ was added followed by slow addition of $p$ - 
toluenesulfonyl chloride $(0.23 \mathrm{~g}, 1.12 \mathrm{mmol})$. The reaction mixture was brought to $\mathrm{RT}$ and stirring continued till the disappearance of compound $8 \mathrm{~b}$ (TLC). The reaction was extracted with $\mathrm{CH}_{2} \mathrm{Cl}_{2}$ and washed with water, organic layer dried $\left(\mathrm{Na}_{2} \mathrm{SO}_{4}\right)$ and solvent evaporated under reduced pressure to give a crude mass, which was purified by column $\left(\mathrm{SiO}_{2}, 60-120\right.$ mesh) using a gradient of EtOAc/Hexane as eluent to give the title compound as a white solid, yield $68 \%$, $0.44 \mathrm{~g}, \mathrm{mp} 68-70{ }^{\circ} \mathrm{C} ; R_{\mathrm{f}} 0.6$ (5:5, Hexane-EtOAc); $[\alpha]_{\mathrm{D}}{ }^{25}-17\left(\mathrm{c} 0.1, \mathrm{CHCl}_{3}\right) ; \mathrm{IR}\left(v_{\max }, \mathrm{cm}^{-1}\right)$ : $3088(\mathrm{~N}-\mathrm{H}), 1640(\mathrm{C}=\mathrm{C}), 1220$ and $770 .{ }^{1} \mathrm{H}$ NMR $\left(300 \mathrm{MHz}, \mathrm{CDCl}_{3}\right): \delta_{\mathrm{H}} 2.04(12 \mathrm{H}, \mathrm{m}, 4 \times-$ $\left.\mathrm{OCOCH}_{3}\right), 2.19\left(3 \mathrm{H}, \mathrm{s},-\mathrm{CH}_{3}\right), 2.68(1 \mathrm{H}, \mathrm{m}, \mathrm{H}-1 \mathrm{~b}), 3.02\left(1 \mathrm{H}, \mathrm{dd}, J_{l} .8 .5 \mathrm{~Hz}, J_{2} .16 .2 \mathrm{~Hz}, \mathrm{H}-1 \mathrm{a}\right)$, $3.73\left(1 \mathrm{H}, \mathrm{m}, \mathrm{H}-1^{\prime}\right), 4.10\left(3 \mathrm{H}, \mathrm{m}, \mathrm{H}-6^{\prime},-\mathrm{NH}\right), 4.29\left(1 \mathrm{H}, \mathrm{m}, \mathrm{H}-5^{\prime}\right), 4.99$ (1H, t, J.9.6 Hz, H-2'), $5.10\left(1 \mathrm{H}, \mathrm{t}, J .9 .8 \mathrm{~Hz}, \mathrm{H}-4^{\prime}\right), 5.25(1 \mathrm{H}, \mathrm{t}, J .9 .3 \mathrm{~Hz}, \mathrm{H}-3$ ') $, 6.70(1 \mathrm{H}, \mathrm{d}, J .16 .1 \mathrm{~Hz}, \mathrm{H}-3), 7.26(4 \mathrm{H}$, m, Ar-H), $7.38(1 \mathrm{H}, \mathrm{m}, \mathrm{Ar}-\mathrm{H}), 7.46(2 \mathrm{H}, \mathrm{m}, \mathrm{H}-4, \mathrm{Ar}-\mathrm{H}), 7.71(1 \mathrm{H}, \mathrm{d}$, J.7.6 Hz, Ar-H), $7.83(1 \mathrm{H}$, d, J.7.9 Hz, Ar-H). ${ }^{13} \mathrm{C} \mathrm{NMR}\left(50 \mathrm{MHz}, \mathrm{CDCl}_{3}\right): \delta_{\mathrm{C}} 20.6\left(4 \times-\mathrm{OCOCH}_{3}\right), 29.6\left(\mathrm{CH}_{3}\right), 42.8(\mathrm{C}-1)$, 61.9 (C-6'), 68.4 (C-2'), 71.6 (C-4'), 74.1 (C-3'), 75.7 (C-1'), 76.2 (C-5'), 120.3 (Ar-C), 122.9 (Ar-C), 126.8 (C-3), 127.2 (Ar-C), 128.6 (Ar-C), 129.6 (Ar-C), 135.4 (Ar-C), 136.2 (Ar-C), 137.7 (Ar-C), 142.5(Ar-C), 143.7 (Ar-C), 144.9 (C-4), 169.2, 169.6, 169.9, 170.4 (4 × - $\mathrm{COCH}_{3}$ ), $195.7(\mathrm{C}=\mathrm{O})$; HRMS: Calcd. Accurate mass for $\left(\mathrm{C}_{31} \mathrm{H}_{35} \mathrm{NNaO}_{12} \mathrm{~S}\right): 668.1778$. Found 668.1765 $[\mathrm{M}+\mathrm{Na}]^{+}$.

(E)- $N$-[3-[4-(1'-Deoxy-2',3',4',6'-tetra- $O$-acetyl- $\beta$-D-glucopyranos-1'-yl)-3-oxobut-1-enyl]phenyl]-2-carbamoylbenzoic acid (14). Solution of the above compound $8 \mathrm{~b}(0.5 \mathrm{~g}, 1.01 \mathrm{mmol})$ and phthalic anhydride $(0.15 \mathrm{~g}, 1.01 \mathrm{mmol})$ in acetone $(15.0 \mathrm{~mL})$ was stirred magnetically at ambient temperature till the disappearance of starting material (TLC). The reaction mixture was extracted with $\mathrm{CH}_{2} \mathrm{Cl}_{2}$ and washed with water, organic layer dried $\left(\mathrm{Na}_{2} \mathrm{SO}_{4}\right)$ and solvent evaporated under reduced pressure to give a crude mass, which was purified by column $\left(\mathrm{SiO}_{2}\right.$, 60-120 mesh) using a gradient of EtOAc/Hexane as eluent to give the compound 14 as a white solid, yield 71\%, $0.46 \mathrm{~g}$, mp 182-184 ${ }^{\circ} \mathrm{C} ; R_{\mathrm{f}} 0.1\left(2: 8\right.$, Hexane-EtOAc); $[\alpha]_{\mathrm{D}}{ }^{25}-27\left(\mathrm{c} 0.1, \mathrm{CHCl}_{3}\right)$; IR $\left(v_{\max }, \mathrm{cm}^{-1}\right): 3520(-\mathrm{COOH}), 3462(\mathrm{~N}-\mathrm{H}), 1639,1216$ and $764 .{ }^{1} \mathrm{H}$ NMR $\left(300 \mathrm{MHz}, \mathrm{CDCl}_{3}\right)$ : $\delta_{\mathrm{H}} 1.95\left(12 \mathrm{H}, \mathrm{m}, 4 \times-\mathrm{COCH}_{3}\right), 2.73(1 \mathrm{H}, \mathrm{m}, \mathrm{H}-4 \mathrm{~b}), 2.95(1 \mathrm{H}, \mathrm{m}, \mathrm{H}-4 \mathrm{a}), 3.74\left(2 \mathrm{H}, \mathrm{m}, \mathrm{H}-1^{\prime}\right.$, $\mathrm{NH}), 4.00$ (1H, m, H-5'), 4.12 (2H, m, H-6'), 4.96-4.82 (2H, m, H-4', H-2'), 5.20 (1H, t, J.9.2 Hz, H-3'), 6.75 (1H, d, J.15.4 Hz, H-2), 7.51 (4H, m, Ar-H), 7.79 (2H, m, Ar-H), 8.05 (2H, m, H-1, $\mathrm{Ar}-\mathrm{H}), 8.36(1 \mathrm{H}, \mathrm{m}, \mathrm{Ar}-\mathrm{H}), 11.45(1 \mathrm{H}, \mathrm{bs},-\mathrm{OH}) .{ }^{13} \mathrm{C} \mathrm{NMR}\left(50 \mathrm{MHz}, \mathrm{CDCl}_{3}\right): \delta_{\mathrm{C}} 20.6(4 \times-$ $\left.\mathrm{OCOCH}_{3}\right), 42.6(\mathrm{C}-4), 62.0\left(\mathrm{C}^{\prime} 6^{\prime}\right), 68.5\left(\mathrm{C}-2^{\prime}\right), 71.7\left(\mathrm{C}-4^{\prime}\right), 73.8\left(\mathrm{C}-3^{\prime}\right), 74.0\left(\mathrm{C}-1^{\prime}\right), 75.3\left(\mathrm{C}-5^{\prime}\right)$, 112.1 (Ar-C), 119.7 (Ar-C), 123.5 (Ar-C), 126.3 (C-2), 128.2 (Ar-C), 129.2 (Ar-C), 129.4 (ArC), 129.9 (Ar-C), 130.3 (Ar-C), 130.5 (Ar-C), 134.7 (Ar-C), 140.4 (Ar-C), 143.4 (C-1), 168.1 ($\mathrm{COOH}), 169.2,169.4,169.6,170.0\left(4 \times-\mathrm{COCH}_{3}\right), 171.7(-\mathrm{NHCO}), 195.8(\mathrm{C}=\mathrm{O})$; HRMS: Calcd. Accurate mass for $\left(\mathrm{C}_{32} \mathrm{H}_{33} \mathrm{NNaO}_{13}\right)$ : 662.1850. Found 662.1853 [M+Na] ${ }^{+}$.

(E)-4-[3-(3-Phenylureido)phenyl]-1-[1'-deoxy-2',3',4',6'-tetra- $O$-acetyl- $\beta$-D-glucopyranos-1'yl]but-3-en-2-one (15). To a stirring solution of compound $8 \mathrm{~b}(0.5 \mathrm{~g}, 1.01 \mathrm{mmol})$ and phenyl isocyanate $(0.12 \mathrm{ml}, 1.12 \mathrm{mmol})$ in $\mathrm{CH}_{2} \mathrm{Cl}_{2}(15.0 \mathrm{~mL}), \mathrm{Et}_{3} \mathrm{~N}(20 \mathrm{~mol} \%)$ was added and stirring continued at ambient temperature till the disappearance of the starting sugar. The reaction mixture was extracted with $\mathrm{CH}_{2} \mathrm{Cl}_{2}$ and washed with water, organic layer dried $\left(\mathrm{Na}_{2} \mathrm{SO}_{4}\right)$ and 
solvent evaporated under reduced pressure to give a crude mass, which was purified by column $\left(\mathrm{SiO}_{2}, 60-120 \mathrm{mesh}\right)$ using a gradient of EtOAc/hexane as eluent to give compound 15 as a yellow solid, yield $69 \%, 0.42 \mathrm{~g}, \mathrm{mp} 85-87^{\circ} \mathrm{C} ; R_{\mathrm{f}} 0.6\left(5: 5\right.$, Hexane-EtOAc); $[\alpha]_{\mathrm{D}}{ }^{25}-18(\mathrm{c} 0.1$, $\left.\mathrm{CHCl}_{3}\right)$; IR $\left(v_{\max }, \mathrm{cm}^{-1}\right): 3425(\mathrm{~N}-\mathrm{H}), 1748(\mathrm{C}=\mathrm{O}), 1621(\mathrm{C}=\mathrm{C}), 1545,1220$ and $771 .{ }^{1} \mathrm{H} \mathrm{NMR}$ $\left(300 \mathrm{MHz}, \mathrm{CDCl}_{3}\right): \delta_{\mathrm{H}} 2.02\left(12 \mathrm{H}, \mathrm{m}, 4 \times-\mathrm{OCOCH}_{3}\right), 2.64(1 \mathrm{H}, \mathrm{m}, \mathrm{H}-1 \mathrm{~b}), 2.90(1 \mathrm{H}, \mathrm{m}, \mathrm{H}-1 \mathrm{a})$, $3.69\left(1 \mathrm{H}, \mathrm{m}, \mathrm{H}-1^{\prime}\right), 4.09$ (3H, m, H-5', $\left.2 \times-\mathrm{NH}\right), 4.28\left(2 \mathrm{H}, \mathrm{m}, \mathrm{H}-6^{\prime}\right), 4.96\left(1 \mathrm{H}, \mathrm{t}, J .9 .5 \mathrm{~Hz}, \mathrm{H}-2^{\prime}\right)$, $5.07\left(1 \mathrm{H}, \mathrm{t}, J .9 .5 \mathrm{~Hz}, \mathrm{H}-4^{\prime}\right), 5.22(1 \mathrm{H}, \mathrm{t}, J .9 .2 \mathrm{~Hz}, \mathrm{H}-3$ ') $, 6.60(1 \mathrm{H}, \mathrm{d}, J .16 .1 \mathrm{~Hz}, \mathrm{H}-3), 7.02(1 \mathrm{H}$, m, Ar-H), $7.11(1 \mathrm{H}, \mathrm{m}, \mathrm{Ar}-\mathrm{H}), 7.23(3 \mathrm{H}, \mathrm{m}, \mathrm{Ar}-\mathrm{H}), 7.31(1 \mathrm{H}, \mathrm{m}, \mathrm{Ar}-\mathrm{H}), 7.37(2 \mathrm{H}, \mathrm{m}, \mathrm{H}-4, \operatorname{Ar}-$ $\mathrm{H}), 7.82(2 \mathrm{H}, \mathrm{m}, \mathrm{Ar}-\mathrm{H}) .{ }^{13} \mathrm{C} \mathrm{NMR}\left(50 \mathrm{MHz}, \mathrm{CDCl}_{3}\right): \delta_{\mathrm{C}} 20.6\left(4 \times-\mathrm{OCOCH}_{3}\right), 42.6(\mathrm{C}-1), 62.0$ (C-6'), 68.4 (C-2'), 71.6 (C-4'), 74.1 (C-3'), 75.7 (C-1'), 76.3 (C-5'), 119.2 (Ar-C), 120.3 (Ar-C), 122.2 (Ar-C), 123.4 (Ar-C), 123.7 (Ar-C), 126.2 (C-3), 129.1 (Ar-C), 129.5 (Ar-C), 134.9 (ArC), 138.2 (Ar-C), 139.2 (C-4), 143.3 (Ar-C), 153.6 (-NHCONH-), 169.3, 169.7, 170.0, 170.6 (4 $\left.\times-\mathrm{COCH}_{3}\right), 196.1(\mathrm{C}=\mathrm{O})$; HRMS: Calcd. Accurate mass for $\left(\mathrm{C}_{31} \mathrm{H}_{34} \mathrm{~N}_{2} \mathrm{NaO}_{11}\right): 633.2060$. Found $633.2047[\mathrm{M}+\mathrm{Na}]^{+}$.

\section{Acknowledgements}

Ramakrishna, Arya and Anindra are thankful to CSIR and UGC New Delhi for the award of JRF and SRF. Financial assistance from DRDO New Delhi and CSIR (THUNDER) New Delhi is gratefully acknowledged. We sincerely thank SAIF division, CDRI for providing the spectral data and microanalyses. This is a CDRI communication 8287.

\section{Supplementary material}

Supplementary data associated with this article can be found, in the online version.

\section{References}

1. Tafesh, A. M.; Weiguny, J. Chem. Rev. 1996, 96, 2035.

2. Downing, R. S.; Kunkeler, P. J.; Van Bekkum, H. Catal. Today 1997, 37, 121.

3. Ono, N. The Nitro Group in Organic Synthesis; Wiley-VCH: New York, 2001.

4. Adams, J. P. J. Chem. Soc., Perkin Trans. 1 2002, 2586.

5. Rylander, P. In Catalytic Hydrogenation in Organic Synthesis; Academic Press: New York, 1979; p 112.

6. Baumeister, P.; Studer, M.; Roessler, F. In Handbook of Heterogeneous Catalysis; Ertl, G., Knözinger, H., Weitkamp, J., Eds.; Wiley-VCH: Weinheim, 1997; pp 2186-2209. 
7. Blaser, H. U.; Siegrist, U.; Steiner, H.; Studer, M. In Fine Chemicals through Heterogeneous Catalysis; Sheldon, R. A., Van Bekkum, H., Eds.; Wiley-VCH: Weinheim, 2001; p 389.

8. Clardy, J.; Walsh, C. Nature 2004, 432, 829.

9. Chau, M.; Jennewein, S.; Walker, K.; Croteau, R. Chem. Biol. 2004, 11, 663.

10. Hubbard, B. K.; Walsh, C. T. Angew. Chem. Int. Ed. 2003, 42, 730.

11. Schwecke, T.; Aparicio, J.; Molnar, I.; Konog, A.; Khaw, L. E.; Haydock, S. F.; Oliynyk, M.; Caffrey, P.; Cortes, J.; Lester, J. B.; Bhom, G. A.; Staunton, J.; Leadlay, P. F. Proc. Natl. Acad. Sci. USA 1995, 92, 7839.

12. Baycroft, B. W. Dictionary of Antibiotics and Related Substances; Chapman and Hall: London, 1988.

13. Faul, M. M.; Huff, B. E. Chem. Rev. 2000, 100, 2407.

14. Faulkner, D. J. Nat. Prod. Rep. 2000, 17, 7.

15. Hacksell, U.; Davies, G. D. Jr. Prog. Med. Chem. 1985, 22, 1.

16. Franz, G.; Grun, M. Planta Med. 1983, 47, 131.

17. Suzuki, K.; Matsumoto, T. In Recent Progress in the Chemical Synthesis of Antibiotics and Related Microbial Products; Lukacs, G., Ed.; Springer: Berlin, 1993; Vol. 2, pp 352-403.

18. Bisht, S. S.; Fatima, S.; Tamrakar, A. K.; Rahuja, N.; Jaiswal, N.; Srivastava, A. K.;Tripathi, R. P. Bioorg. Med. Chem. Lett. 2009, 19, 2699.

19. Bisht, S. S.; Jaiswal, N.; Sharma, A.; Fatima, S.; Sharma, R.; Rahuja, N.; Srivastava, A. K.; Bajpai, V.; Kumar, B.; Tripathi, R. P. Carbohydr. Res. 2011, 346, 1191.

20. Compain, P.; Martin, Q. R. BioOrg. Med. Chem. 2001, 9, 3077 and reference cited therein.

21. Lai, H. L.; Axelrod, B. BioChem. Biophys. Res. Commun. 1973, 54, 463.

22. Winchester, B.; Fleet, G. W. J. Glycobiology 1992, 2, 199.

23. Durette, P. L.; Bugianesi, R. L.; Ponpipom, M. M.; Shen, T. Y.; Cascieri, M. A.; Glitzer, M. S.; Katzen, H. M. J. Med. Chem. 1978, 21, 854.

24. Trost, B. M. Science 1983, 219, 245.

25. Burns, N. Z.; Baran, P. S.; Hoffmann, R. W. Angew. Chem. Int. Ed. 2009, 48, 2854.

26. Young, I. S.; Baran, P. S. Nature Chem. 2009, 1, 193.

27. Trost, B. M.; Dong, G. B. Nature 2008, 456, 485.

28. Afagh, N. A.; Yudin, A. K. Angew. Chem. Int. Ed. 2010, 49, 262-310.

29. Rai, G.; Jeong, J. M.; Lee, Y. S.; Kim, H. W.; Lee, D. S.; Chung, J. K.; Lee, M. C. Tetrahedron Lett. 2005, 46, 3987.

30. Bates, D. K.; Li, K. J. Org. Chem. 2002, 67, 8662.

31. Khan, F. A.; Dash, J.; Sudheer, C.; Gupta, R. K. Tetrahedron Lett. 2003, 44, 7783.

32. Deshpande, R. M.; Mahajan, A. N.; Diwakar, M. M.; Ozarde, P. S.; Chaudhari, R. V. J. Org. Chem. 2004, 69, 4835.

33. Saha, A.; Ranu, B. J. Org. Chem. 2008, 73, 6867.

34. Noronha, R. G.; Romao, C. C.; Fernandes, A. C. J. Org. Chem. 2009, 74, 6960. 
35. Gamble, A. B.; Garner, J.; Gordon, C. P.; O'Conner, S. M. J.; Keller, P. A. Synthetic Commun. 2007, 37, 2777.

36. Bisht, S. S.; Pandey, J.; Sharma, A.; Tripathi, R. P. Carbohydr. Res., 2008, 343, 1399.

37. Rodrigues, F.; Canac, Y.; Lubineau, A. Chem. Commun. 2000, 2049.

38. Riemann, I.; Papadopoulos, M. A.; Knorst, M.; Fessner, W. D. Aust. J. Chem. 2002, 55, 147.

39. Wang, J. F.; Lei, M.; Li, Q.; Ge, Z.; Wang, X.; Li, R. Tetrahedron 2009, 65, 4826.

40. Barros, Ana I. R. N. A.; Silva, A. M. S. Tetrahedron Lett. 2003, 44, 5893.

41. Barros, Ana I. R. N. A.; Dias, A. F. R.; Silva, A. M. S. Monatsh. Chem. 2007, 138, 585. 